\title{
Multi-scale image based pore space characterisation and pore network generation: case study of a north sea sandstone reservoir
}

\author{
Gilbert Scott · Kejian Wu • Yingfang \\ Zhou
}

Received: date / Accepted: date

\begin{abstract}
In this paper we examine the pore space geometry and topology of a North Sea sandstone reservoir rock based on multi-scale scanning electron microscopy (SEM). The reservoir was subjected to extensive diagenesis which has resulted in a complex pore space with a wide range in pore sizes. We quantify the pore size and pore coordination number distributions and we find that the mean and standard deviation of the coordination number are power law functions of pore radius, where the scaling exponent varies from 0.3 to 0.5 . We present a $2 \mathrm{D}$ stochastic algorithm to generate a pore network based on statistical information. The algorithm incorporates the concept of a weighted planar stochastic lattice which is a construction that naturally leads to scale-free character with power law behaviour. We validate the algorithm against SEM imaging by showing that it can reproduce the observed clustering and a realistic spatial distribution of pore space elements. We also try to explore the relationship between fluid flow properties of reservoir rock and $2 \mathrm{D}$ pore image features.
\end{abstract}

Keywords porous media $\cdot$ pore network $\cdot$ SEM imaging $\cdot$ multi-scale

\section{Introduction}

Over the last 20 years there have been substantial advances in many pore scale imaging, characterisation and modelling techniques (Blunt et al., 2013; Bultreys et al., 2016). One of the key goals of these techniques is to obtain pore structure information and determine macroscopic properties such as porosity, permeability, capillary pressure and relative permeability based on the microscopic structural information and physical properties of the fluids. Pore scale models of natural porous media are increasingly being used for engineering purposes in the evaluation of oil and gas resources and environmental engineering such as water resource management and carbon dioxide storage.

There has been considerable success in the prediction of multi-phase flow properties (capillary pressure and relative permeability) for homogeneous rocks with

School of Engineering, University of Aberdeen, Aberdeen, AB24 3UE, UK E-mail: r01ggs16@abdn.ac.uk 
narrow pore size distributions such as Berea or Fontainebleau sandstones (Øren et al., 1998; Patzek, 2001). However, more complex sandstones containing substantial cements, clays and grain dissolution (secondary porosity) typically have much wider pore size distributions and still remain a significant challenge (Bashtani et al., 2016; Lai et al., 2018).

Complex sandstones typically have a long geological history and have been deeply buried (2000-5000 m). The porosity and pore space topology is strongly influenced by diagenetic processes such as carbonate cementation, quartz overgrowths and pore filling clays (e.g. kaolinite, illite and chlorite). Porosity is reduced by compaction, cementation and authigenic clays. Diagenesis typically creates smaller and more disconnected pores (Cook et al., 2011). The combination of multiple, complex processes results in a wide range in pore sizes (Lai et al., 2018). The traditional concepts of pore body and pore throat can be difficult to apply in complicated reservoir rocks.

In our work, we have used multi-scale SEM for 2D imaging and X-ray micro computed tomography (micro-CT) for 3D imaging (Bultreys et al., 2016). All technologies have a trade-off between resolution and field of view, but this can be more of a problem for micro-CT imaging since it has a more limited resolution and sample volume. We used micro-CT to image a 3D volume of about $1 \mathrm{~mm}^{3}$ at circa $1 \mu \mathrm{m} /$ voxel. In comparison, our SEM images cover an area of up to $6 \mathrm{~mm}^{2}$ at $0.2 \mathrm{\mu m} /$ pixel. SEM imaging is also faster and lower cost than micro-CT, hence a larger number of samples can be analysed within the same time frame and budget. High resolution is important to image the smallest pore features but a large field of view is also important to capture the largest pores.

Although it is possible to study pore space morphology and extract pore space models directly from 3D micro-CT volumes (Silin and Patzek, 2006; Lindquist et al., 1996; Rabbani et al., 2014), such models are limited by the inherent resolution and field of view of the data. To incorporate high resolution information from SEM imaging, a number of authors have developed voxel based 3D reconstruction algorithms based on 2D training images (Manwart et al., 2000; Keehm et al., 2004; Wu et al., 2004; Tahmasebi and Sahimi, 2012; Naraghi et al., 2017). These methods still have a limitation in that an unfeasibly large number of voxels will be required to capture 3 to 4 orders of magnitude variation in pore sizes.

Many carbonate systems also have a wide range of pore sizes (Youssef et al., 2007; De Boever et al., 2012; Freire-Gormaly et al., 2015). Typically, these porous media exhibit multiple porosity and multi-modal pore size distributions e.g. macropores created during primary deposition and micropores created by subsequent partial dissolution or cementation. A number of approaches for generating multiscale pore space models have been proposed for these systems (Békri et al., 2005; Okabe and Blunt, 2007; Jiang et al., 2013; Bultreys et al., 2015). All of these methods essentially create networks at different scales from different resolution images and then join them together in some way. In sandstones, the combination of multiple diagenetic processes can create a wide range of pore sizes without necessarily being multi-modal. Accordingly, we seek an efficient continuous modelling methodology that avoids making distinctions at different scales.

Instead of linking different models at different scales or generating a very large high resolution image and extracting its pore network, we propose to construct a stochastic pore network directly using statistical information from high resolution images. In principle, such a process can incorporate information from multiple $2 \mathrm{D}$ 
and $3 \mathrm{D}$ images at a range of resolutions. High resolution 2D imaging can be used to capture pore size information and connectivity information from $2 \mathrm{D}$ can be validated against 3D micro-CT data (at a lower resolution). Also, Rabbani et al. (2016) have published an empirical correlation between 2D and 3D coordination numbers for isotropic porous media. This would suggest it is possible to estimate the $3 \mathrm{D}$ connectivity function from $2 \mathrm{D}$ information. Having said this, we do not think that multiscale SEM imaging is a replacement for 3D micro-CT imaging. The techniques are complementary, with SEM imaging providing superior multiscale pore size information and micro-CT imaging providing superior $3 \mathrm{D}$ connectivity information.

A number of authors have published stochastic pore network generation algorithms. These generally fall into two categories. Firstly, algorithms that (implicitly or explicitly) assume the pore space is simple and the pore size distribution is not too wide (Idowu and Blunt, 2008; Jiang et al., 2012). These algorithms honour connectivity information such as the coordination number distribution or the connectivity function (Vogel and Roth, 2001) but they assume that pore bodies (network nodes) are uniformly distributed in space. These algorithms have successfully constructed networks that reproduced multi-phase flow functions for simple homogeneous sandstones. The second group of algorithms are based on the concept of heterogeneous preferential attachment (HPA) (Santiago, 2008; Yakubo and Koroak, 2011) which is an extension of the Barabasi-Albert (BA) model of heterogeneous networks (Barabasi and Albert, 1999). These algorithms are fundamentally modelling heterogeneous networks and they lead to the emergence of scale-free character with a power law behaviour. The HPA concept results in networks where the probability of coordination number $Z$ is $P(Z) \propto Z^{\alpha}$ where $\alpha$ is a scaling exponent. Although these models have been applied to complex soil systems and successfully reproduce observed power-law behaviour, it has not been demonstrated that they can reproduce multi-phase flow behaviour.

Our stochastic algorithm is inspired by the weighted planar stochastic lattice (WPSL) (Hassan et al., 2010) which is a structure displaying scale-free character with power law behaviour but we also incorporate explicit connectivity information as per Jiang et al. (2012). The algorithm has some similarities with the multiplicative cascade algorithm used to model fractures (Verscheure et al., 2012). The WPSL algorithm is detailed below.

In this work, we present a pore space characterisation case study for a North Sea sandstone reservoir that was buried to depths up to $3000 \mathrm{~m}$ and subjected to extensive diagenesis. The principal geological formations are the Upper Jurassic Fulmar sandstone and the Triassic Skagerrak. The Fulmar formation is a highly bioturbated shallow marine sandstone divided into three main units (upper, middle and lower) and comprising of two main facies classes (Cannon and Gowland, 1996). The first facies class is bioturbated, silty sandstones and the second facies class is bioturbated, coarse grained sandstones deposited in a high energy wave/current environment. The Skagerrak consists of red sandstones and shales deposited in a fluvial and alluvial environment. The main reservoir intervals are upwards fining laminated sand channels, ranging from clean coarse grained at the base to fine grained muddy sands at the top (Akpokodje et al., 2017).

Figure 1 shows a scanning electron microscope (SEM) image of a sample from the Skagerrak formation. Cements and clays are evident plus secondary porosity created by partial grain dissolution. Figure 2 shows mercury injection capillary 
pressure (MICP) data from three different core plug samples. Typically, samples contain a range of pore throat sizes of 2 to 3 orders of magnitude.

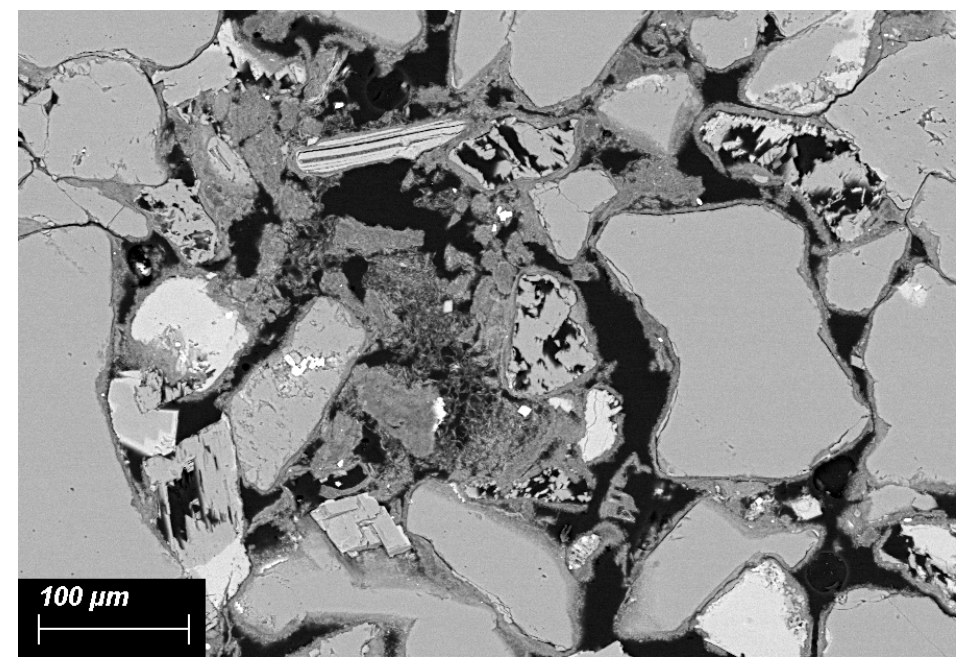

Fig. 1 SEM image of Skagerrak sandstone sample

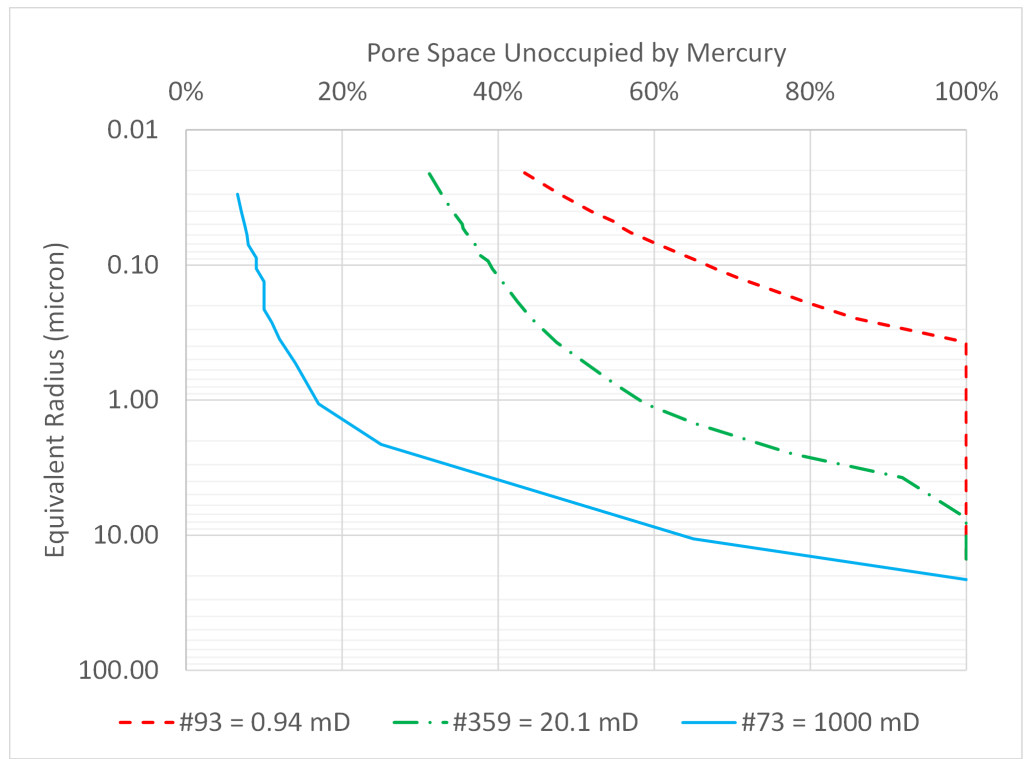

Fig. 2 MICP data from three core plug samples

In this paper we discuss the characterisation results from $2 \mathrm{D}$ imaging and the stochastic pore network algorithm in $2 \mathrm{D}$. The extension to $3 \mathrm{D}$ and prediction of multi-phase flow properties will be addressed in future work. 


\section{Multi-Scale Image Acquisition and Processing}

\subsection{Acquisition}

Ten core plug samples were selected for analysis and one polished block was created for each sample. The samples were selected to capture a representative range of porosity and permeability for each zone with reference to a cross-plot of permeability vs porosity for all available samples (Figure 3). The sample selection was biased towards the Skagerrak and Lower Fulmar because these zones are much thicker than the Upper and Middle Fulmar. No rigorous statistical analysis was performed to select the samples.

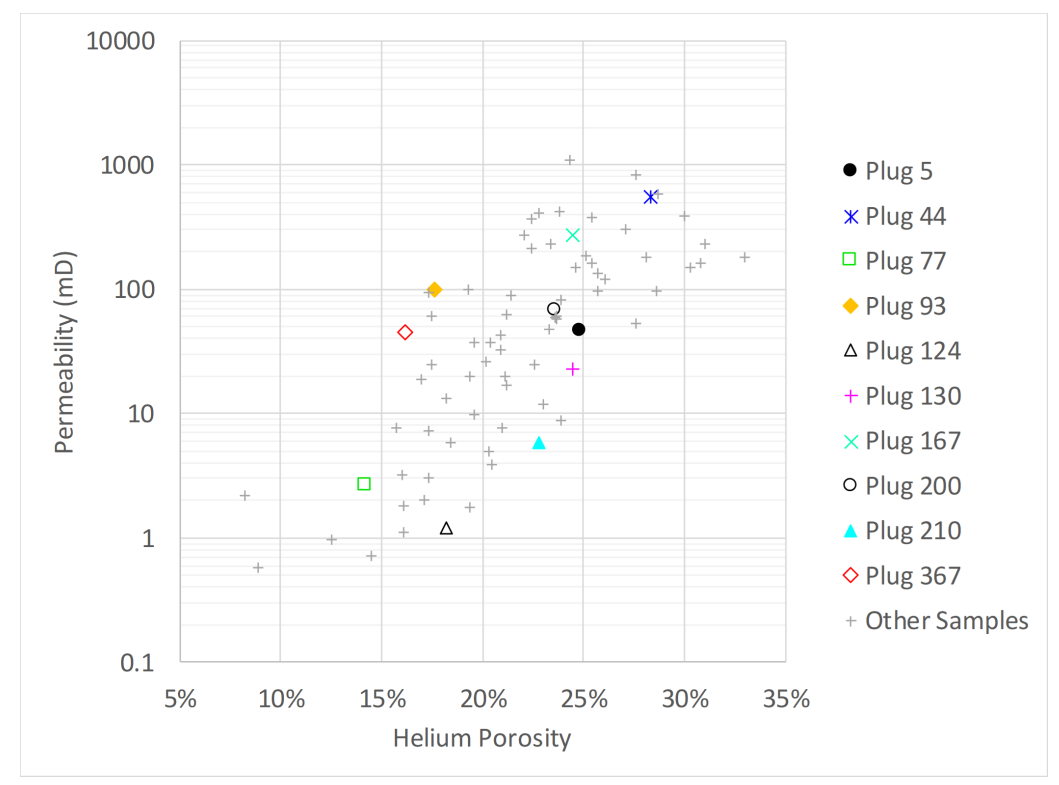

Fig. 3 Cross plot of measured permeability and porosity for all plug samples

A subsample of each core plug was cut, this was injected with resin under vacuum and then polished until optically flat. The polished blocks were imaged with two instruments: Topcon ABT-60 SEM and Carl Zeiss GeminiSEM 300. All SEM images were acquired at multi-scale from the backscattered electron (BSE) detector.

Basic properties of the samples are given in Table 1. Porosity, permeability and capillary pressure were not measured as part of this work. These properties were measured previously and the data provided by the project sponsor with the samples. Porosity was measured by helium porosimeter, permeability was measured by nitrogen permeameter and the data were Klinkenberg corrected by the laboratory. The laboratory report does not provide any information on experimental errors. The precision of 3 significant figures is consistent with the data presented in the laboratory report. The variation between samples from the same zone is a reflection of geological heterogeneity. 
Macro porosities were determined from air-brine capillary pressure or MICP data where macropores (micropores) are defined as those larger (smaller) than 0.2 $\mu \mathrm{m}$. Note that, for the low permeability samples (77 and 124), around two-thirds of the pore space is classified as microporosity which cannot be imaged by micro-CT or the high resolution SEM used in this work. This would require higher resolution SEM to reveal the micropore information.

Table 1 Rock sample properties; macro porosity is obtained from capillary pressure measurements and means pores larger than $0.2 \mu \mathrm{m}$

\begin{tabular}{|c|c|c|c|c|}
\hline Sample & Zone & $\begin{array}{c}\text { Helium } \\
\text { Porosity }\end{array}$ & $\begin{array}{c}\text { Macro } \\
\text { Porosity }\end{array}$ & $\begin{array}{c}\text { Permeability } \\
(\mathrm{mD})\end{array}$ \\
\hline 5 & Upper Fulmar & $24.8 \%$ & $16.2 \%$ & 46 \\
44 & Middle Fulmar & $28.3 \%$ & $22.7 \%$ & 557 \\
77 & Lower Fulmar & $14.2 \%$ & $5.3 \%$ & 2.7 \\
93 & Middle Fulmar & $17.6 \%$ & $13.6 \%$ & 100 \\
124 & Lower Fulmar & $18.2 \%$ & $5.3 \%$ & 1.2 \\
130 & Lower Fulmar & $24.5 \%$ & $16.7 \%$ & 23 \\
167 & Skagerrak & $24.5 \%$ & $19.1 \%$ & 270 \\
200 & Skagerrak & $23.6 \%$ & $15.8 \%$ & 68 \\
210 & Skagerrak & $22.8 \%$ & $12.5 \%$ & 5.8 \\
367 & Skagerrak & $16.2 \%$ & $10.4 \%$ & 45 \\
\hline
\end{tabular}

Two multi-scale SEM imaging strategies were employed in this work. Firstly, all ten samples were imaged using the Topcon ABT-60 SEM at a range of magnifications with the same image size $(1400 \times 862$ pixels $)$. Generally, four images were captured at each of four magnifications, corresponding to spatial resolutions of $2.12,1.06,0.53$ and $0.27 \mu \mathrm{m}$ per pixel. This is a rapid process as each image can be captured in less than two minutes. These images were used in a downscaling algorithm which progressively adds detail from higher magnification images into the lowest magnification image to result in a final image with a high spatial resolution and a wide field of view. Details of the downscaling algorithm are given in Appendix A. The downscaling was applied twice for each sample to generate two final images based of different intermediate images. The final downscaled images have a spatial resolution of $0.27 \mu \mathrm{m}$ per pixel and an image size of $8800 \times 6400$ pixels.

Secondly, a single wide field, high resolution image was acquired for each of five samples using the Carl Zeiss GeminiSEM 300. These images have a spatial resolution of $0.17 \mu \mathrm{m}$ per pixel and an image size of $16384 \times 12288$ pixels. The acquisition time for each image was about 3 hours as they have a large size.

\subsection{Segmentation}

The greyscale SEM images were segmented by applying a threshold. We investigated Otsu's method (Otsu, 1979) of segmentation, but this quite often resulted in porosity greater than the measured helium porosity. Instead, the segmentation threshold was determined by taking a fraction of the interval between two peaks of the pixel intensity histogram. An example for plug 367 is shown in Figure 4. The first intensity peak corresponds to the pore space (near black) and the second 
peak corresponds to the quartz matrix (light grey). The selected threshold was equal to the intensity of the first peak plus $10 \%$ of the interval between the first and second peaks. For the downscaled images, which have lower image resolution, the threshold was the intensity of the first peak plus $30 \%$ of the interval between the peaks.

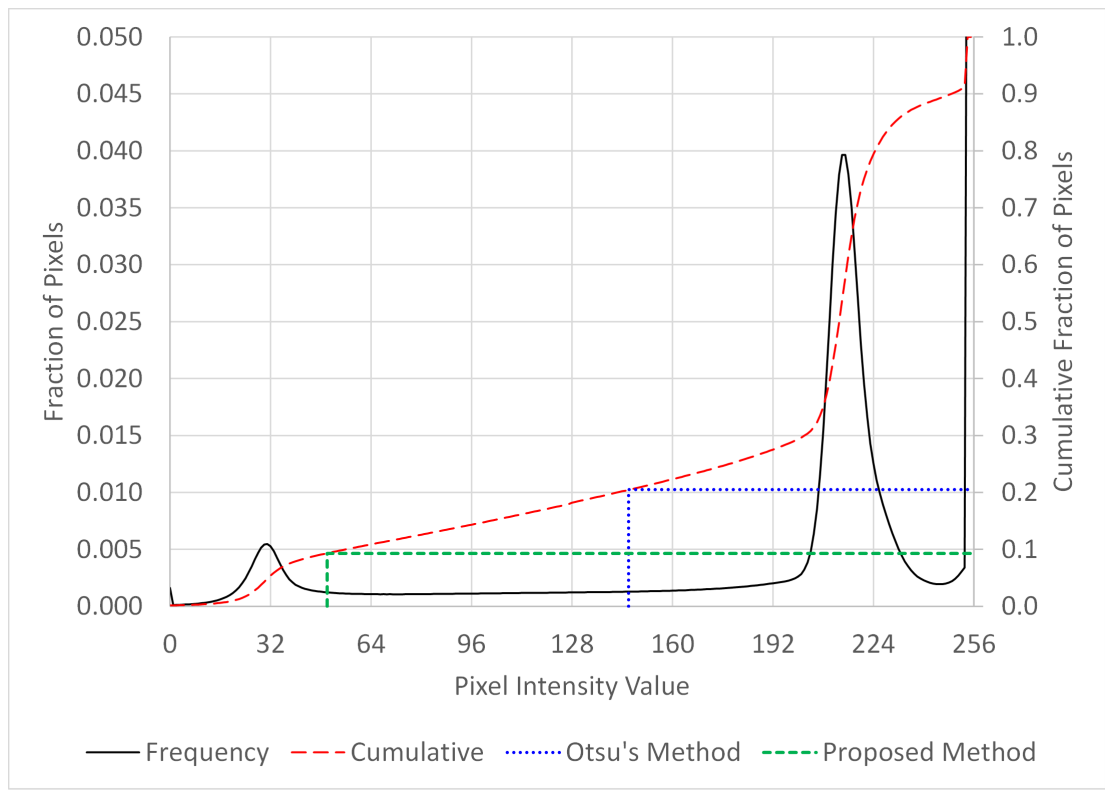

Fig. 4 Plug 367 high resolution image pixel intensity frequency histogram and cumulative intensity, showing threshold and resulting porosity from (i) Otsu's method and (ii) the proposed method

There was some residual noise after segmentation, therefore we applied a filter to remove isolated pixel clusters (islands) of 4 pixels or less. The filter removes both small black areas (isolated porosity) and small white areas (floating particles). Small pores which are connected are retained. The filter substantially reduces the number of isolated pixel clusters with minimal impact on the porosity. Note that the filter preserves information below $1 \mu \mathrm{m}$ and is not equivalent to removing pores of diameter 4 pixels or less because those features are likely to be rounded and will consist of more than 4 pixels.

Figure 5 compares the porosity from SEM imaging with the macro porosity from capillary pressure data. Generally, we find there is agreement within \pm 3 porosity units (PU). The capillary pressure data were measured on whole core plug samples whereas the SEM images cover a much smaller area, therefore the differences in porosity estimates may indicate some heterogeneity at the core plug scale. 


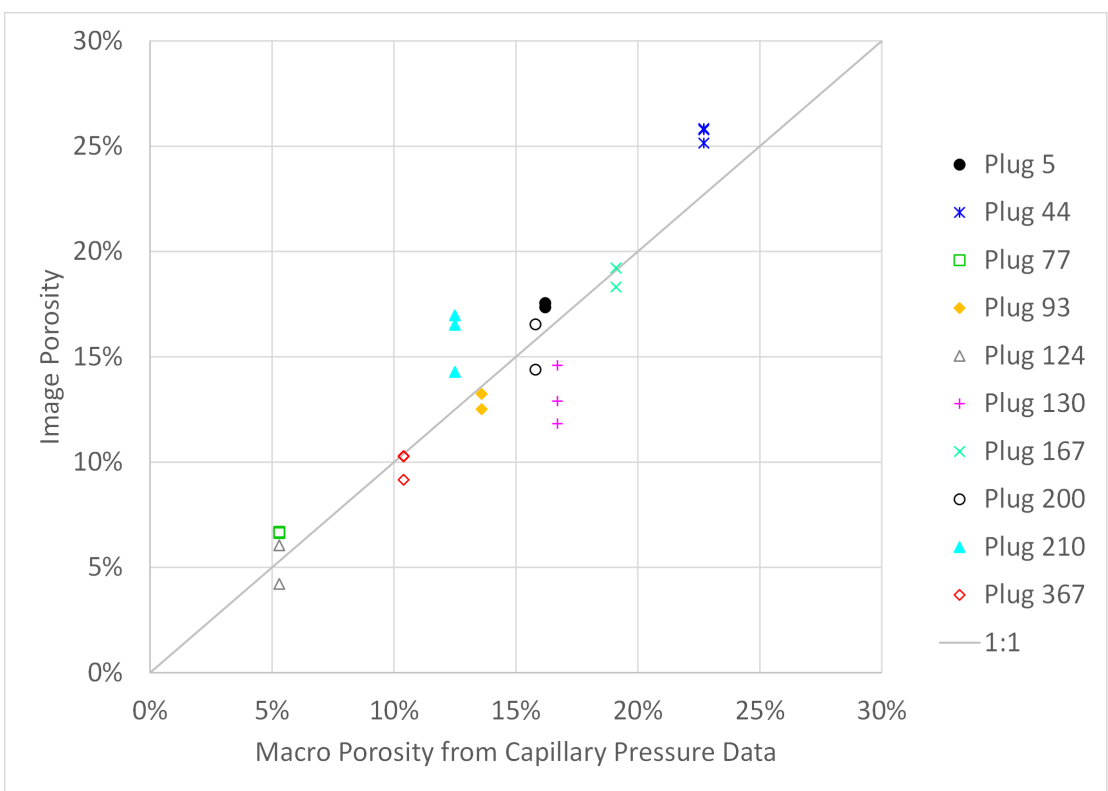

Fig. 5 Porosity from SEM image analysis vs macro porosity from capillary pressure data

\section{Pore Space Characterisation}

\subsection{Methodology}

In this work we have characterised the pore space by discretising it into elements and performing a statistical analysis of the elements. The definition of a pore space element is a little arbitrary, but each element is based on an inscribed circle which touches the pore wall in at least two separate places (generally on opposite sides). The Euclidean distance map is the Euclidean distance from each pore space pixel to the nearest solid (rock matrix) pixel and the medial axis is the set of points which have more than one closest point on the pore wall. A circle centered on the medial axis with radius equal to the Euclidean distance at its center will touch the pore wall in at least two separate places. Therefore we consider the centers of the pore elements to be constrained to the medial axis and we define the pore element radius from the value of the Euclidean distance map at the center of the element.

The boundaries between elements are approximately perpendicular to the medial axis of the pore space and the links between element centres capture the pore space topology. We consider links between elements because we wish to characterise the connectivity of the pore space for flow modelling. The methodology assumes that the principal direction of flow is parallel to the medial axis. The elements are identified in sequence from largest to smallest such that largest pore bodies are captured first and represented by a minimal number of elements.

The process of discretising the pore space is conceptually similar to extracting a pore network from imaging data. Therefore, we could use an existing pore network extraction algorithm such as the maximal ball method (Silin and Patzek, 2006), a medial axis method (Lindquist et al., 1996) or a watershed algorithm 
(Rabbani et al., 2014). However, we have potentially large and complex images to analyse, therefore we have designed a discretisation algorithm that incorporates elements from existing methodologies but most importantly is pragmatic and efficient. Starting from a segmented binary image, the algorithm is summarised as follows:

1. Calculate the Euclidean distance map of the pore space.

2. Calculate the ridge points of the Euclidean distance map.

3. Construct a set of inscribed circles centered on the ridge points.

4. Assign each pixel to a pore space element.

5. Identify the connections between pore space elements.

Figure 6 illustrates the process for a simple test case.

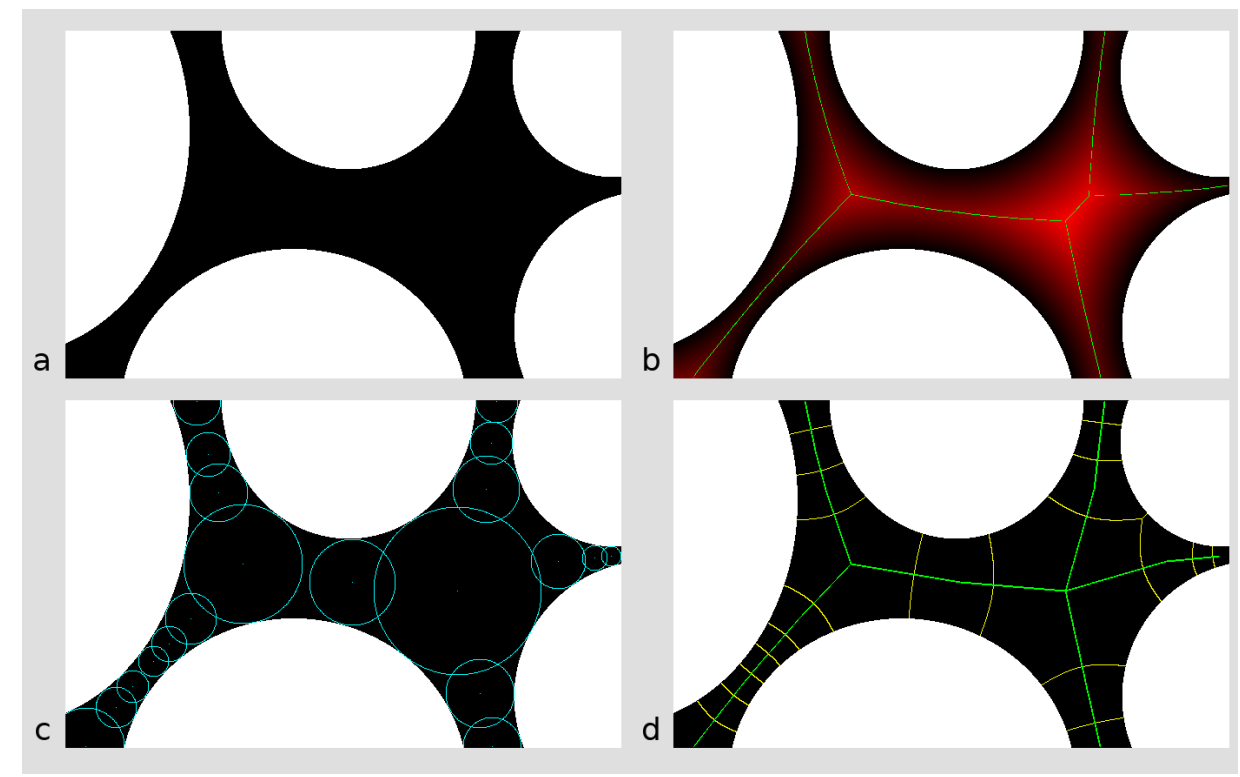

Fig. 6 Pore space discretisation: (a) binary image with pore space in black (b) Euclidean distance map in red shading and ridge points in green (c) inscribed circles centered on ridge points (d) boundaries between pore space elements in yellow and connections between elements in green

To calculate the Euclidean distance map, we use an efficient algorithm due to Borgefors (1984).

Next we identify the ridge points of the Euclidean distance map which is an approximation of the skeleton or medial axis. We use a very efficient, straightforward criterion which defines a ridge point based on approximating the curvature or second derivative of the Euclidean distance. In particular, a pixel is defined as a ridge point if it satisfies:

$$
8 E_{i}-\sum_{j=1}^{8} E_{j} \geqslant 3
$$


Where $E_{i}$ is the Euclidean distance of pixel $i$ and the sum is over the eight neighbours of the pixel. The value 3 is used because this is required to identify the ridge points in a rectangular area with side length equal to an even number of pixels. Note that for a general shape, the ridge points are not necessarily continuous and single pixel width. Figure $6 \mathrm{~b}$ highlights the ridge points in green.

From here, we limit the search to the identified ridge points. We find the maximum value of the Euclidean distance map, construct a circle of radius equal to that Euclidean distance and then remove those ridge points inside the circle plus an extra tolerance (1.25 times the circle radius). Then find the next largest value of the Euclidean distance map and repeat the process until there are no remaining ridge points. At this point the pore space is covered by circles although there can be both gaps and overlaps (Figure 6c). The balance between overlap and gaps is controlled by the tolerance factor. If the factor is greater than 2 , there is guaranteed to be no overlap and if the factor is less than 1 there will be no gaps. Based on the images we have analysed, we find that a value of 1.25 generally achieves a reasonable compromise with, on average, the distance between circle centers being close to the sum of the radii.

Now we partition the pore space and assign each pixel to a pore space element. To do this we start at the centre of each element and search all connected pixels to a maximum distance of three times the element radius. Each pixel is assigned to the element with the smallest value of $E_{i j}-r_{j}$ where $E_{i j}$ is the Euclidean distance from pixel $i$ to the center of element $j$ and $r_{j}$ is the radius of element $j$. This criterion ensures that:

1. where circles overlap, the boundary between elements passes through the intersection points, and

2. where there is a gap, the boundary between elements passes through the mid point of the shortest line connecting the circles' circumferences.

Boundaries of pore space elements are shown in yellow in Figure 6d.

Finally, we identify connections between pore space elements based on the interface between pixel regions belonging to different elements. A connection is created between two elements if the interface length is greater than the harmonic mean of their radii. This criterion ensures that small interfaces do not create spurious connections that would not honour the topology. Connections between elements are shown in green in Figure 6d.

Figure 7 shows the pore space discretisation of extracts from three SEM images.

\subsection{Results}

Figure 8 shows the pore size distribution from the high resolution images.

A comparison between the high resolution and downscaled images for plug 130 is shown in Figure 9.

Here, we are plotting the complementary cumulative probability distribution $\Phi(r)=\operatorname{Pr}(R>r)$ as a function of $r$. The high resolution images have a better spatial resolution than the downscaled images, the minimum resolved pore size is smaller and therefore we see the pore size distributions shifted to the left. Accounting for this effect, there is good qualitative agreement between distributions from the two SEM imaging strategies. 


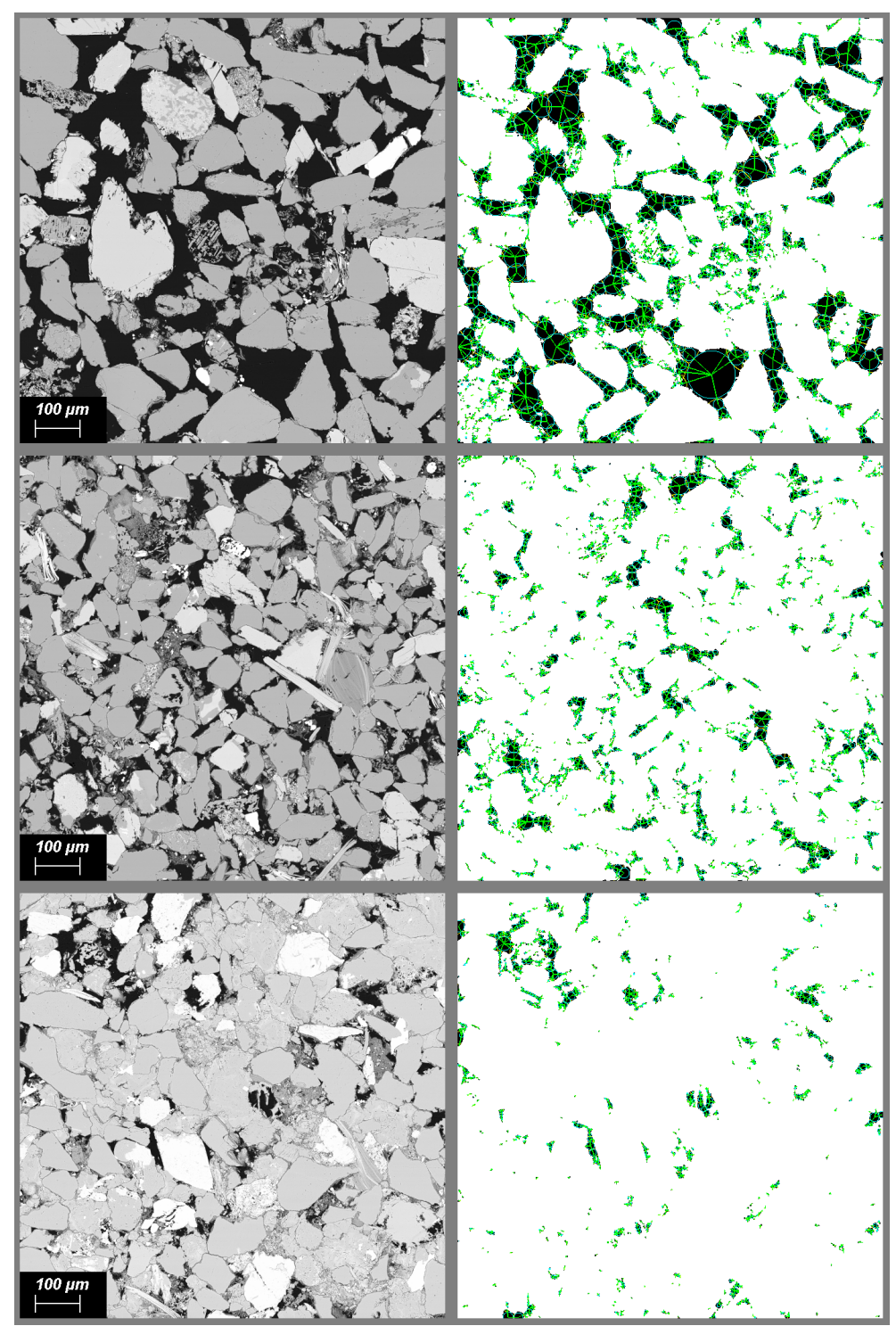

Fig. 7 Pore space characterisation. SEM image (left) and pore space element discretisation of binary image (right). Plug 44 (top), plug 130 (middle) and plug 77 (bottom).

In all cases, we observe a linear relationship between $\log (\Phi(r))$ and $\log (r)$ at small radii (Pareto Type I distribution) with an exponential cut-off at larger radii. We have fitted the data to a cumulative probability distribution function of the form:

$$
\Phi(r)=\left(\frac{r}{r_{0}}\right)^{\alpha} \exp \left(-\left(\frac{r}{r_{1}}\right)^{n}\right)
$$

Where $\alpha$ is the slope parameter or scaling index, $r_{0}$ is a parameter controlling the lower limit of the distribution, $r_{1}$ controls the position of the cut-off and $n$ controls 


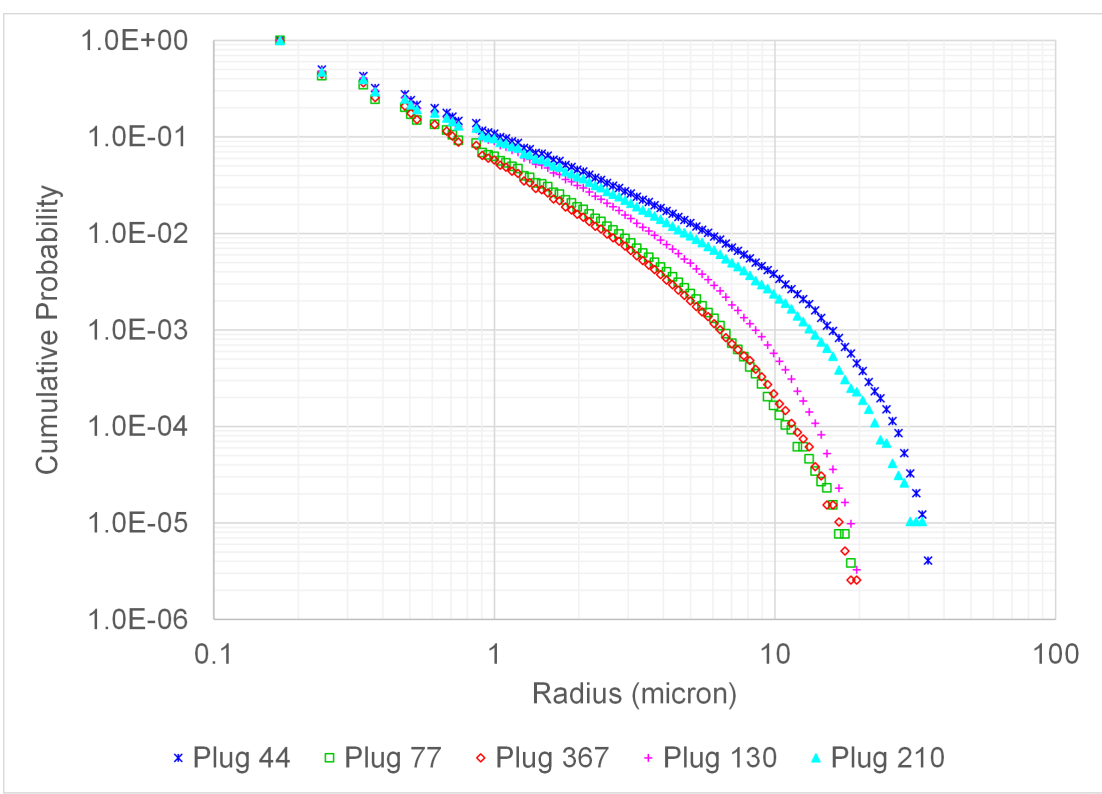

Fig. 8 Cumulative pore size distribution from high resolution SEM images

the shape of the cut-off. The fitted parameters are given in Tables 2 and 3. The final columns in these tables give a measure of goodness of fit in terms of the root mean square error (RMSE) of the logarithm of the cumulative probability.

The differences between the pore size distributions of different samples are due to geological variations in grain size, silt content, cementation and clay content. Grain size is a primary control on permeability and porosity is controlled by cementation and silt content (Cannon and Gowland, 1996; Gowland, 1996; Akpokodje et al., 2017). Within the Fulmar, the Middle unit was deposited in the highest energy environment and has the largest grain size. Samples 44 and 93 from the Middle Fulmar exhibit larger pore sizes consistent with lower silt and clay content. Samples 77 and 124 from the Lower Fulmar have smaller pore sizes consistent with smaller grain size, higher silt content and a lower energy depositional environment (Gowland, 1996).

Table 2 High resolution images: pore size distribution parameters

\begin{tabular}{|c|c|c|c|c|c|}
\hline Sample & $\begin{array}{c}r_{0} \\
\text { (micron) }\end{array}$ & $\begin{array}{c}r_{1} \\
\text { (micron) }\end{array}$ & $\alpha$ & $n$ & RMSE \\
\hline 44 & 0.172 & 14.5 & 1.25 & 1.84 & 0.040 \\
77 & 0.172 & 2.86 & 1.32 & 0.94 & 0.049 \\
130 & 0.172 & 5.54 & 1.31 & 1.31 & 0.049 \\
210 & 0.172 & 13.2 & 1.33 & 1.88 & 0.070 \\
367 & 0.172 & 5.78 & 1.59 & 1.34 & 0.060 \\
\hline
\end{tabular}

Figure 10 shows a plot of the slope parameters $\alpha$ from all images. We see that the downscaled images give systematically lower slope parameter. We suspect this 


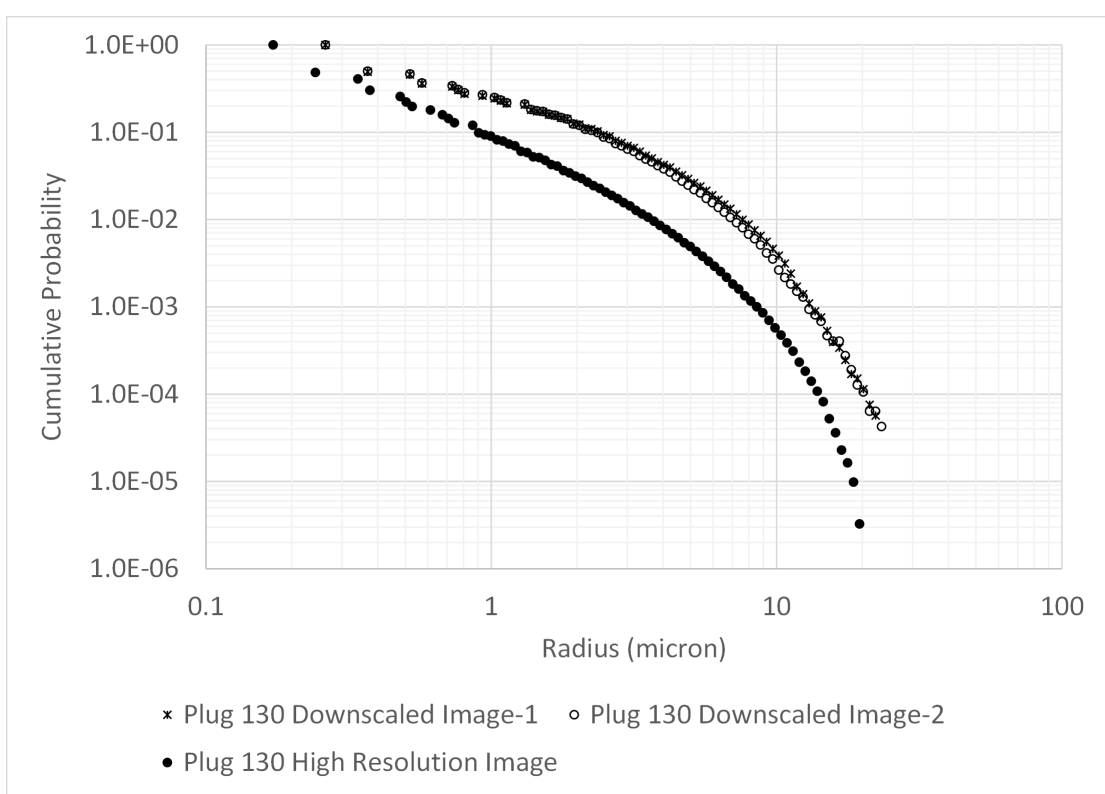

Fig. 9 Comparison of pore size distribution from high resolution and downscaled SEM images: plug 130

Table 3 Downscaled images: pore size distribution parameters

\begin{tabular}{|c|c|c|c|c|c|c|}
\hline Sample & Image & $\begin{array}{c}r_{0} \\
\text { (micron) }\end{array}$ & $\begin{array}{c}r_{1} \\
\text { (micron) }\end{array}$ & $\alpha$ & $n$ & RMSE \\
\hline 5 & 1 & 0.270 & 7.45 & 1.09 & 1.05 & 0.072 \\
5 & 2 & 0.270 & 7.52 & 1.08 & 1.14 & 0.062 \\
44 & 1 & 0.270 & 15.80 & 1.01 & 1.24 & 0.032 \\
44 & 2 & 0.270 & 15.17 & 0.99 & 1.29 & 0.029 \\
77 & 1 & 0.270 & 5.09 & 1.04 & 1.22 & 0.063 \\
77 & 2 & 0.270 & 4.88 & 1.01 & 1.15 & 0.073 \\
93 & 1 & 0.270 & 14.01 & 0.98 & 1.27 & 0.031 \\
93 & 2 & 0.270 & 12.62 & 1.08 & 1.23 & 0.055 \\
124 & 1 & 0.270 & 2.47 & 0.94 & 1.10 & 0.048 \\
124 & 2 & 0.270 & 2.66 & 0.97 & 0.96 & 0.070 \\
130 & 1 & 0.270 & 6.28 & 0.98 & 1.50 & 0.035 \\
130 & 2 & 0.270 & 4.88 & 0.92 & 1.23 & 0.036 \\
167 & 1 & 0.270 & 10.04 & 1.00 & 1.44 & 0.028 \\
167 & 2 & 0.270 & 11.80 & 1.06 & 1.56 & 0.045 \\
200 & 1 & 0.270 & 8.35 & 1.06 & 1.46 & 0.044 \\
200 & 2 & 0.270 & 5.94 & 0.90 & 1.01 & 0.029 \\
210 & 1 & 0.270 & 11.99 & 0.99 & 1.16 & 0.033 \\
210 & 2 & 0.270 & 13.58 & 1.01 & 1.30 & 0.037 \\
367 & 1 & 0.270 & 9.52 & 1.20 & 1.35 & 0.086 \\
367 & 2 & 0.270 & 9.74 & 1.15 & 1.43 & 0.087 \\
\hline
\end{tabular}

is due to imaging limitations and some residual noise in the highest magnification images. These effects result in erroneous extension of the pore size distribution to the smallest radii i.e. parameters $r_{0}$ and $\alpha$ should both be larger. We have more confidence in the high resolution images because they were obtained with a more advanced SEM instrument. 


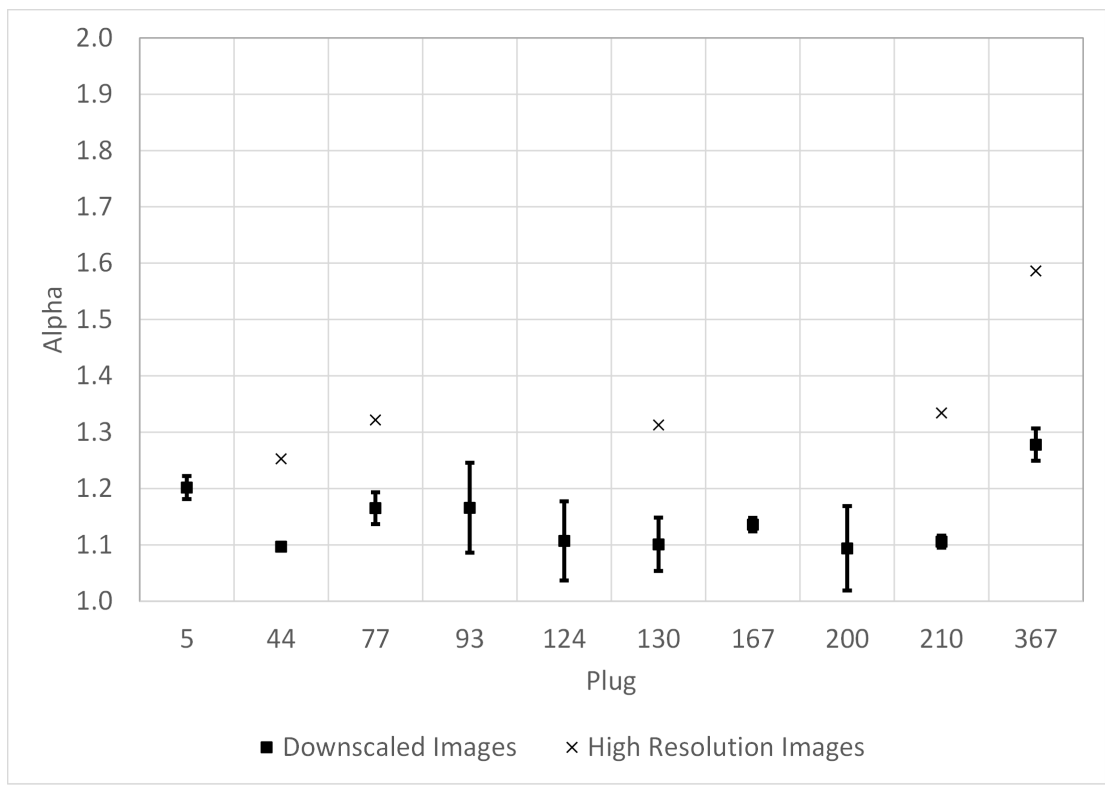

Fig. 10 Slope parameter or scaling index of the pore size distribution for all plug samples

Figure 11 compares the cumulative pore size distribution from image analysis with the pore throat size distribution calculated from drainage air-brine capillary pressure data. For the SEM images, we are simply plotting the cumulative area fraction of pore elements less than a given radius. To make a more valid comparison, we have added the microporosity (radii below $0.1 \mu \mathrm{m}$ ) on to the SEM image analysis distribution. There is fair agreement between the datasets. The image analysis tends to shows larger pore sizes, particularly for plug 77 , this is partly because the drainage capillary pressure is controlled by pore throats and therefore under-estimates the radius associated with a given saturation. Discrepancies could also be explained by heterogeneity within the samples since the capillary pressure was measured for the whole core plug.

To compare SEM image analysis with the measured air permeability of the plug samples we have calculated a radius which is characteristic of the largest pores which control permeability. Katz and Thompson (1987) show that permeability is related to the square of a characteristic length which is close to the size of the largest pores and can be obtained from the inflection point on the capillary pressure curve. Here, we obtain a characteristic radius from the fitted pore size distribution which includes an exponential cut-off which characterises the tail of the distribution. The parameter $r_{1}$ is not suitable for this purpose because it depends on the cut-off shape parameter $n$. Instead, we choose $r_{x}$ to be the radius where the exponential cut-off reduces the cumulative probability to one tenth of the value it would have without the cut-off.

$$
\exp \left(-\left(\frac{r_{x}}{r_{1}}\right)^{n}\right)=0.1
$$

Thus:

$$
r_{x}=r_{1}(-\log (0.1))^{1 / n}
$$




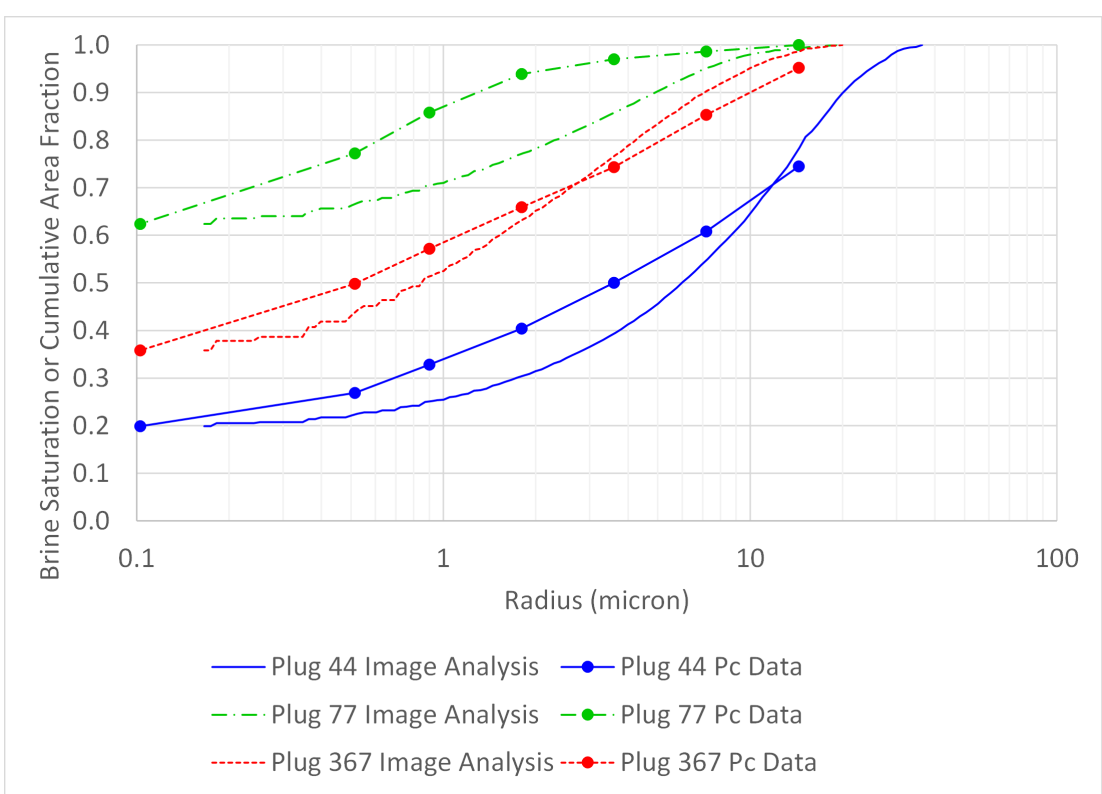

Fig. 11 Pore size distributions from SEM image analysis and air-brine capillary pressure data

The factor of one tenth is arbitrary and does not have any physical significance and therefore, we do not claim to predict permeability directly. Rather, we obtain a parameter that is closely related to permeability.

Figure 12 shows a plot of $\phi r_{x}^{2}$ against the measured air permeability. A clear trend is evident with higher permeability samples having a higher value of $\phi r_{x}^{2}$. Plug 210 is an outlier; the SEM image analysis would suggest that the permeability is higher than has been measured. The image porosity for plug 210 is also higher than expected (Figure 5), therefore we suspect there is some low porosity/permeability heterogeneity within the core plug which is not captured by the SEM imaging.

The frequency distribution of coordination numbers for the high resolution images is shown in Figure 13. For coordination numbers greater than 2, we observe an exponential behaviour where the probability of coordination number $Z$ is $P(Z) \propto \exp (-a Z)$ and the constant $a$ is 0.5 to 0.7 . This is contrary to the HPA type model proposed by Santiago (2008) for soil pore structures, which has a power law behaviour with scale free character.

For a given element radius, we find that the coordination number is close to a normal distribution. The mean and standard deviation of the coordination number increase with radius as shown in Figures 14 and 15.

We have fitted the mean and standard deviation of the coordination number to power law relationships of the form:

$$
Z_{\text {mean }}(r)=A_{\text {mean }} r^{\beta}
$$

and

$$
Z_{\text {stdev }}(r)=A_{\text {stdev }} r^{\beta}
$$




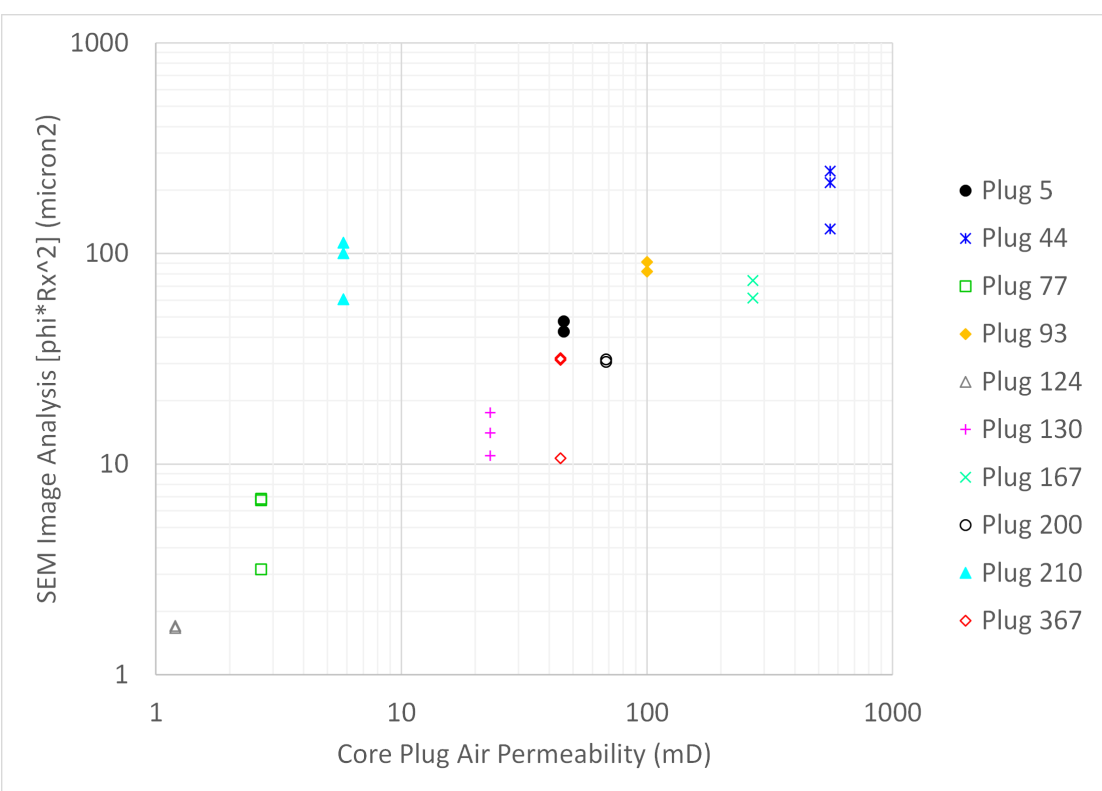

Fig. 12 Parameter $\phi r_{x}^{2}$ from image analysis vs measured core plug air permeability

The overall mean coordination number $Z_{\text {mean }}$, the parameters $A_{\text {mean }}, A_{\text {stdev }}$ and $\beta$ are given in tables 4 and 5 . The final columns in these tables give the root mean square error (RMSE) of the logarithm of the mean coordination number.

Table 4 High resolution images: coordination number parameters

\begin{tabular}{|c|c|c|c|c|c|}
\hline Sample & $Z_{\text {mean }}$ & $A_{\text {mean }}$ & $A_{\text {stdev }}$ & $\beta$ & RMSE \\
\hline 44 & 2.2112 & 3.24 & 1.12 & 0.33 & 0.027 \\
77 & 2.0645 & 3.42 & 1.21 & 0.38 & 0.030 \\
130 & 2.1082 & 3.13 & 1.11 & 0.37 & 0.030 \\
210 & 2.2352 & 3.39 & 1.23 & 0.36 & 0.023 \\
367 & 2.0855 & 3.52 & 1.20 & 0.42 & 0.027 \\
\hline
\end{tabular}

A comparison between the high resolution and downscaled images for plug 130 is shown in Figure 16. There is good agreement in the mean coordination number between the imaging strategies.

\section{Pore Space Modelling}

\subsection{Methodology}

Here, we present a stochastic algorithm to generate pore networks based on the pore space characterisation information above. The motivation for this is to overcome the trade-off between resolution and field of view in micro-CT imaging. In order to represent complex sandstones such as the Fulmar and Skagerrak, it is necessary to capture pore sizes ranging by about three orders of magnitude (e.g. 0.1 


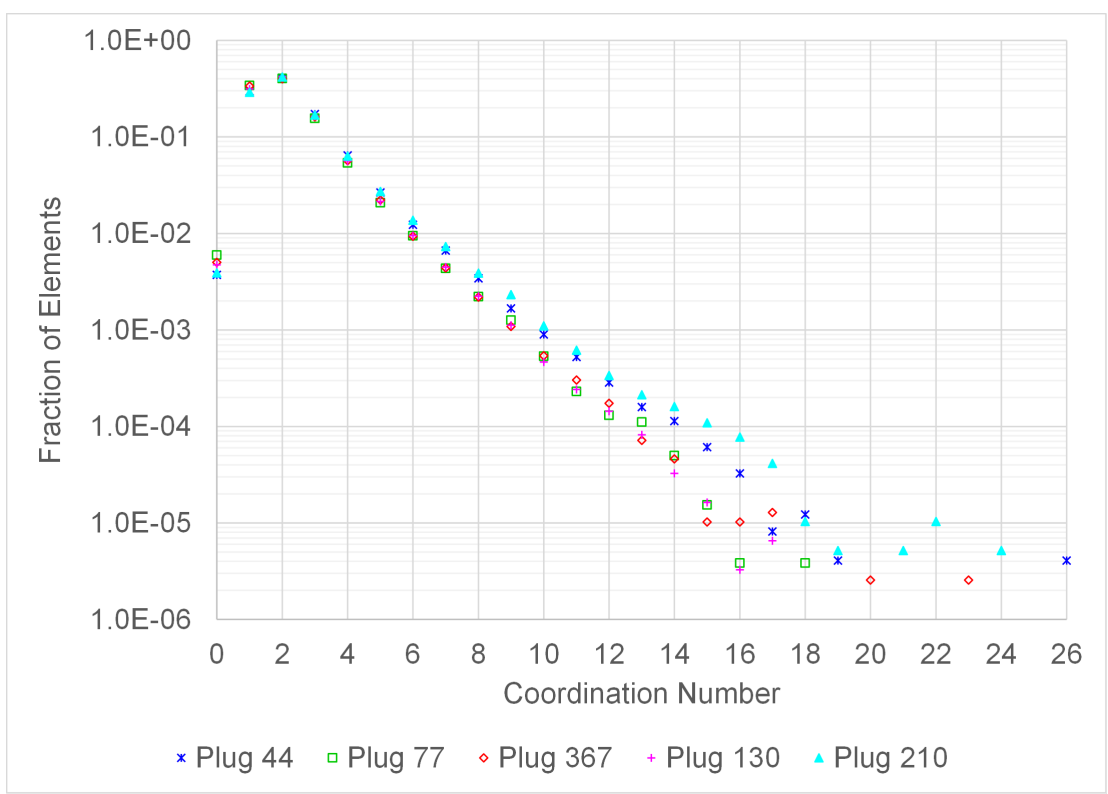

Fig. 13 Coordination number frequency distribution for high resolution images

Table 5 Downscaled images: coordination number parameters

\begin{tabular}{|c|c|c|c|c|c|c|}
\hline Sample & Image & $Z_{\text {mean }}$ & $A_{\text {mean }}$ & $A_{\text {stdev }}$ & $\beta$ & RMSE \\
\hline 5 & 1 & 2.5456 & 3.19 & 1.04 & 0.37 & 0.049 \\
5 & 2 & 2.5577 & 3.20 & 1.03 & 0.39 & 0.036 \\
44 & 1 & 2.5952 & 3.17 & 1.03 & 0.34 & 0.047 \\
44 & 2 & 2.5956 & 3.08 & 1.05 & 0.33 & 0.048 \\
77 & 1 & 2.4715 & 3.09 & 1.09 & 0.40 & 0.041 \\
77 & 2 & 2.4979 & 3.06 & 1.03 & 0.38 & 0.047 \\
93 & 1 & 2.5682 & 3.09 & 1.04 & 0.33 & 0.040 \\
93 & 2 & 2.5444 & 3.05 & 1.02 & 0.37 & 0.033 \\
124 & 1 & 2.4556 & 3.17 & 1.01 & 0.39 & 0.055 \\
124 & 2 & 2.4430 & 3.16 & 1.06 & 0.37 & 0.059 \\
130 & 1 & 2.5251 & 3.05 & 1.00 & 0.37 & 0.030 \\
130 & 2 & 2.5122 & 3.02 & 1.03 & 0.38 & 0.029 \\
167 & 1 & 2.5726 & 3.07 & 1.04 & 0.36 & 0.036 \\
167 & 2 & 2.5673 & 3.05 & 1.03 & 0.36 & 0.037 \\
200 & 1 & 2.5267 & 3.06 & 1.02 & 0.37 & 0.038 \\
200 & 2 & 2.5339 & 3.05 & 1.00 & 0.35 & 0.040 \\
210 & 1 & 2.5709 & 3.06 & 1.05 & 0.33 & 0.046 \\
210 & 2 & 2.5711 & 3.08 & 1.04 & 0.32 & 0.049 \\
367 & 1 & 2.5362 & 3.34 & 1.06 & 0.38 & 0.053 \\
367 & 2 & 2.5252 & 3.22 & 1.07 & 0.35 & 0.051 \\
\hline
\end{tabular}

$\mu \mathrm{m}$ to $100 \mu \mathrm{m}$ ) and the network should cover a representative elementary volume (REV) which is likely at least an order of magnitude larger than the largest pore size i.e. greater than $1 \mathrm{~mm}$. It is challenging to image such a volume at sufficient resolution. Hence, an attractive option is to generate a statistically representative pore network using statistical information from SEM imaging. In principle, such 


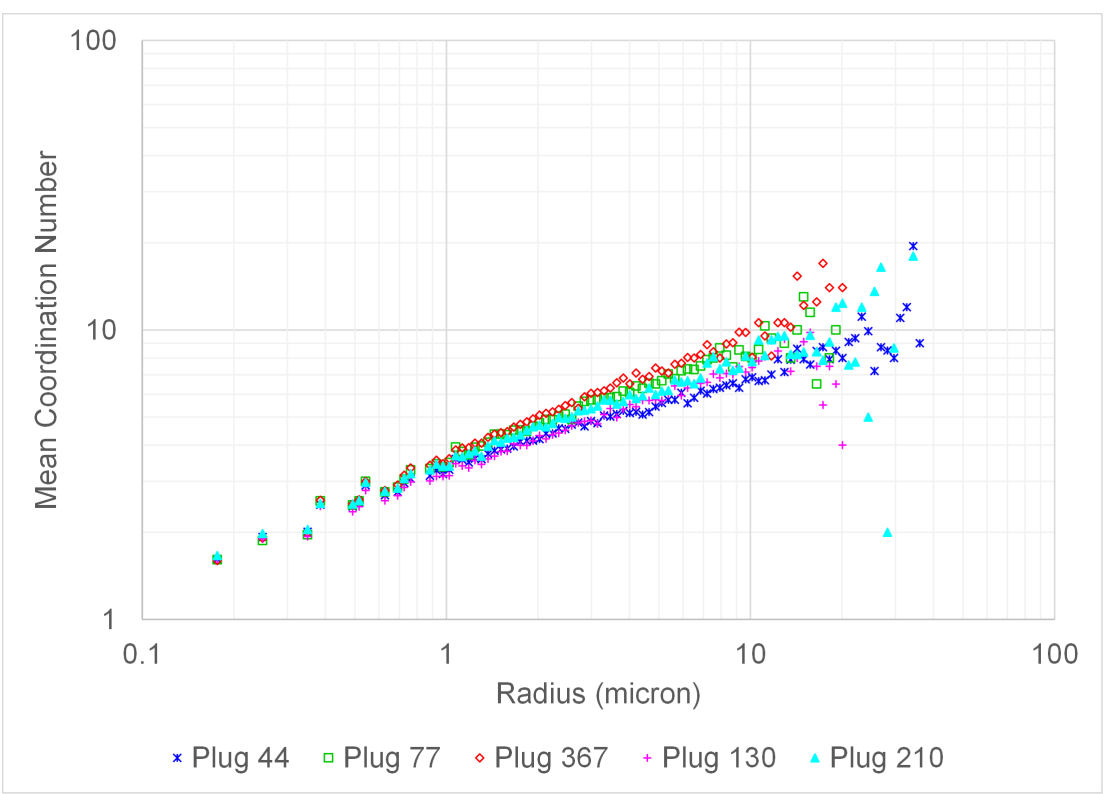

Fig. 14 Mean coordination number as a function of element radius for high resolution images

an algorithm can generate networks of arbitrary size with an arbitrary ranges of pore sizes.

Our stochastic algorithm incorporates some ideas from the weighted planar stochastic lattice (WPSL) (Hassan et al., 2010). A WPSL is a space filling cellular structure on a rectangular domain. To construct a WPSL, we start with a rectangle and apply a generator that randomly divides it into four smaller rectangles. Then we repeatedly apply the generator to one of the available rectangles chosen preferentially with respect to their areas.

The general WPSL can result in many rectangles with extreme aspect ratios, therefore we consider the more specialised construction which is limited to squares. The starting point is a square domain and on each iteration we choose one square at random and divide it into four equal squares. It is clear that the number of squares increases by 3 on each iteration. After any number of iterations we can construct a network from the dual graph i.e. by placing a node at the center of each square and constructing a bond between any two squares which share a common border. Figure 17 shows a simple example.

The WPSL has three key properties that make is attractive as a model for porous media. Firstly, large squares naturally have high coordination numbers and small squares have low coordination numbers. Secondly, the coordination numbers of the general WPSL obey a power law distribution $P(Z) \propto Z^{-\gamma}$ with $\gamma=5.66$ (Hassan et al., 2010). Thirdly, the recursive process naturally fits into multiscale pore structure hierarchies. These, properties are similar to but do not perfectly match our pore space characterisation, therefore we consider a modification of the WPSL where not all squares are occupied by nodes. This enables us to modify the coordination number of pore elements to match characterisation data. 


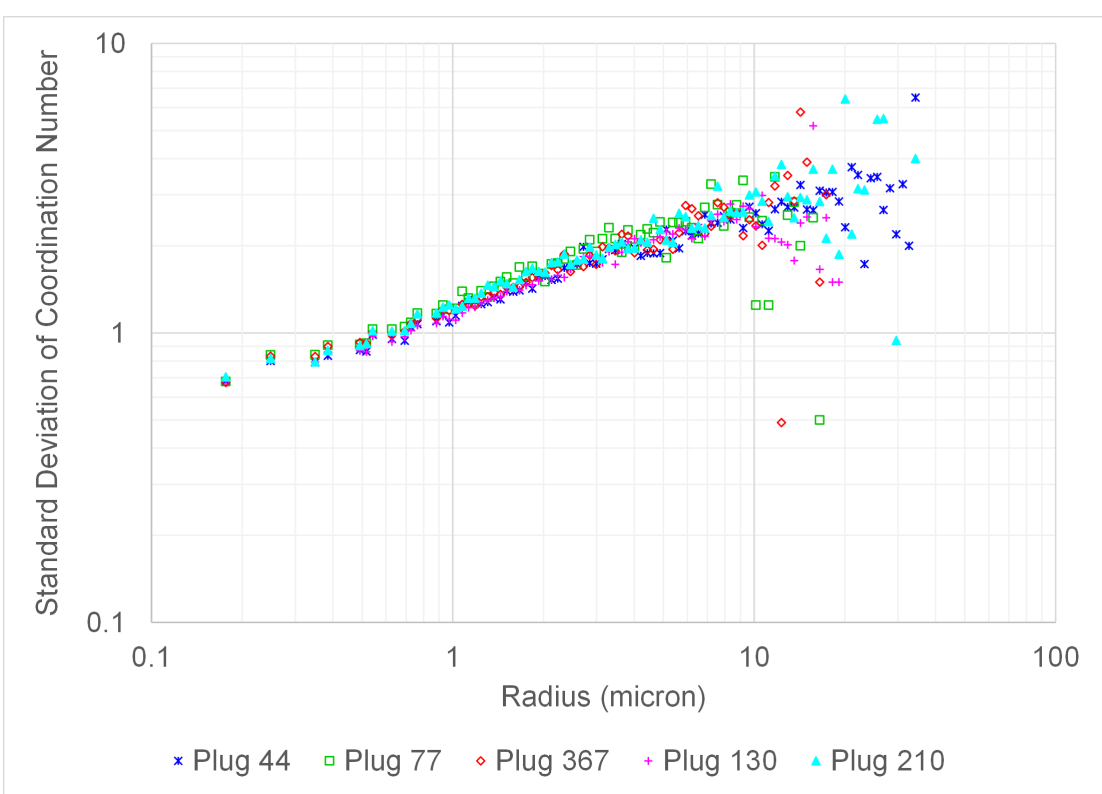

Fig. 15 Standard deviation of the coordination number as a function of element radius for high resolution images

The stochastic algorithm also has some similarities with the multiplicative cascade algorithm (Verscheure et al., 2012). We divide the domain recursively and we assign features from a power law distribution. However, we also wish to explicitly honour connectivity/topological information which is not addressed by Verscheure et al. (2012).

To generate a network representing the pore space of a real complex sandstone, we need to honour some key information. In particular, we honour the pore element size distribution and connectivity information in the form of the pore element coordination number distribution as a function of pore radius. From image analysis, we have also determined the links between elements and we can define the radius of a link as equal to the minimum of the two connected element radii. In this way we can determine the distribution of the link radii.

The connectivity function is known to be important for controlling multi-phase flow behaviour (Vogel and Roth, 2001). Here, it is simple to show that the connectivity function is given by:

$$
\chi_{A}(r)=\frac{N_{e}(r)-N_{l}(r)}{A}
$$

Where $N_{e}(r)$ and $N_{l}(r)$ are the numbers of elements and links with radii greater than $r$ inside an area $A$. Our definition of link radius means that a link with radius greater than $r$ will always be connected to two elements with radii greater than $r$. This means that we can construct a straightforward algorithm that honours the connectivity function as well as the coordination number information.

The algorithm starts with domain of a given size and inserts a given total number of elements into the domain. In this work we have generated networks of 


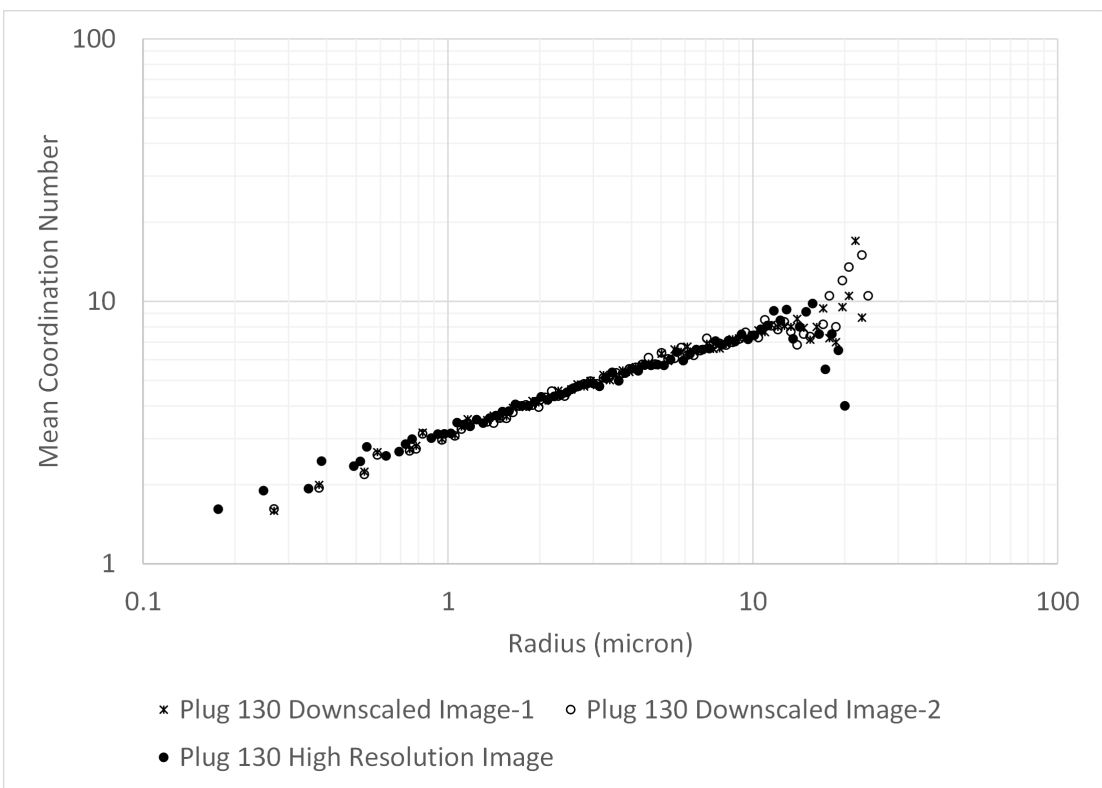

Fig. 16 Comparison of mean coordination number for high resolution and downscaled SEM images: plug 130

size equal to the SEM images from which the statistics were obtained and we know the total number elements from the image analysis. Alternatively, the number of elements could be determined from a target porosity because the distribution of element radii is given and the area of an element is proportional to its area. Analysis of our SEM images shows that the proportionality factor is between 3.7 and 4.0 .

The algorithm takes some ideas from published stochastic pore network generation algorithms. In particular, we start at the largest pore size and progressively add elements into the network in a way that honours the connectivity function as per Jiang et al. (2012). We also assign a target coordination number to each element and decrement the target each time the element is linked to another one as per Idowu and Blunt (2008). Unlike these existing algorithms, we do not assume that pore elements are uniformly randomly distributed in space. As shown below, this is a poor assumption for complex sandstones because the pore elements are actually spatially clustered. This is a key motivation for using the WPSL construction because the WPSL enables smaller pore elements to be located in the proximity of existing (larger) pore elements and linked to them in a systematic way.

The complete stochastic algorithm is as follows:

1. Start with a rectangular domain of given size.

2. Calculate the minimum and maximum pore radii of interest, $r_{\min }$ and $r_{\max }$.

3. Let the given total number of elements be $N_{e, t o t a l}$. Construct an array of $N_{e, t o t a l}$ elements where each element has a radius sampled from the distribution and a target coordination number $z_{i}$ sampled from the distribution based on its radius. 


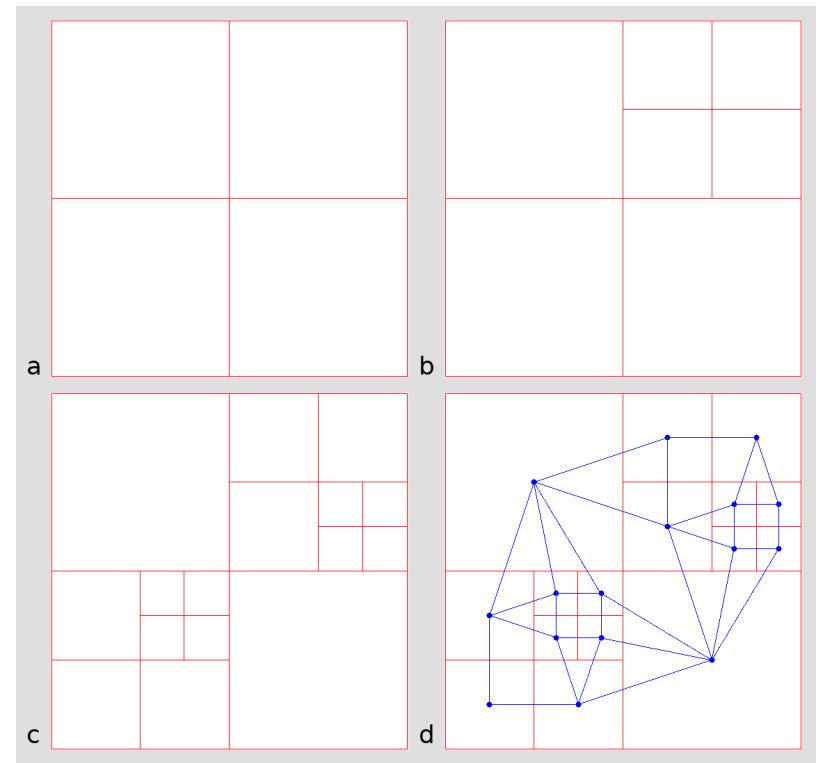

Fig. 17 Weighted planar stochastic lattice limited to square structures: (a) domain divided into four squares (b) after the second iteration (c) after five iterations (d) the resulting network or dual graph in blue

4. Calculate the total number of links $N_{l, \text { total }}=\frac{1}{2} \sum_{i=1}^{N_{e, t o t a l}} z_{i}$ and construct an array of links where each link has a radius sampled from the distribution.

5. Divide the domain into squares of side length $L_{\max }>r_{\max }$.

6. Set $r=r_{\max }$.

7. Calculate the number of elements and links with radii between $r$ and $r / 2$; $N_{e}=N_{e}(r / 2)-N_{e}(r)$ and $N_{l}=N_{l}(r / 2)-N_{l}(r)$.

8. Position the $N_{e}$ elements and $N_{l}$ links in order of radius, starting with the largest. For each element, if there exists a link with a radius smaller than the element but larger than the next smallest element then position this element at the center of a square adjoining an existing (i.e. larger) element chosen at random and assign the link to the pair of elements. If there is no such link, then position the element at the center of a randomly selected square that does not adjoin any existing elements.

9. In the previous step, when we need to position an element adjoining an existing one, the probability of choosing each one of the existing elements is proportional to its target coordination number and when a link is created, the target coordination numbers of the two linked elements are each decreased by one.

10. Divide each of the unoccupied squares into four equal squares.

11. Set $r=r / 2$.

12. If $r>r_{\min }$ go to step 7 .

A simple illustration of the procedure is illustrated in Figure 18.

In step 5 we need to choose a value of $L_{\max }$. It should be clear that the ratio $L_{\max } / r_{\max }$ controls the average overlap or gap between element radii. If 

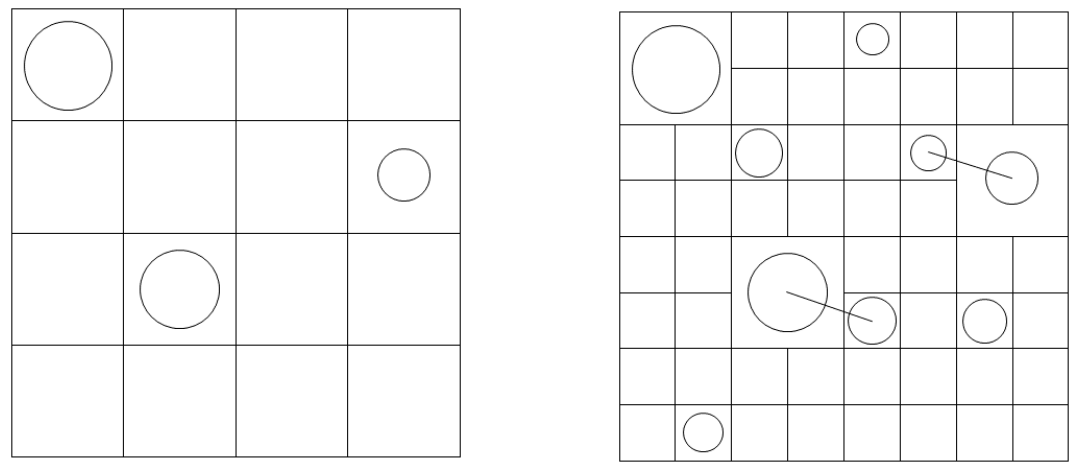

Fig. 18 Stochastic pore network generation: (a) divide the domain into squares and insert elements with radii between $r_{\max }$ and $r_{\max } / 2(\mathrm{~b})$ divide each of the unoccupied squares into four and insert elements with radii between $r_{\max } / 2$ and $r_{\max } / 4$, if there exists a link with radius larger than the next smallest element, then position the element adjacent to an existing one and link them

$L_{\max } / r_{\max }>2$ then there will be no overlap and if $L_{\max } / r_{\max }<1$ there will be no gaps. In the pore space characterisation we used a tolerance factor of 1.25 when deleting ridge points from the Euclidean distance map. To be consistent with this methodology we choose $L_{\max } / r_{\max }=1.25$.

The algorithm proceeds by dividing the pore size distribution into a geometric progression with a common ratio of a half. At each iteration we insert elements of appropriate radii into squares of similar size. Step 8 tries to insert $N_{e}$ elements and $N_{l}$ links at each iteration. For the first few iterations, generally $N_{e}$ is much greater than $N_{l}$ and therefore most large elements are positioned in randomly selected squares not adjoining existing elements. These elements will be linked to smaller elements in later iterations. In the last few iterations, generally $N_{e}$ is less than $N_{l}$ and therefore most small elements are positioned adjacent to existing (larger) elements and linked to them. If an element is, by chance, positioned adjacent to two or more existing elements then links are inserted between all the adjacent elements. In all cases the largest radius unassigned link is used. The algorithm tends to result in the element and link arrays being assigned in parallel which ensures that the connectivity function is honoured. Throughout the process, the radius of the largest unassigned element is close to the radius of the largest unassigned link. This means that the radius of a link will be close to the minimum of the two connected element radii, which is consistent with the definition of the link radius given above. If the link array is exhausted before the element array then the last few elements are positioned without linking to any existing elements. If the element array is exhausted before the link array then the last few links are discarded.

When an element is positioned in a square adjacent to an existing element we need to choose a particular location for the element. If the existing element is linked to zero, two or more other elements, we choose the location at random from the unoccupied squares. However, if the existing element is linked to just one other element, we assign a higher probability to the sites on the opposite side 
of the square. This makes it more likely that the angle between the links will be close to $180^{\circ}$ rather than $90^{\circ}$ or $270^{\circ}$. Using our pore space characterisation of SEM images, we have examined elements that have a coordination number of 2 and measured the angle, $\theta$, between the links. We find that $\operatorname{Pr}\left[135^{\circ}<\theta<225^{\circ}\right]$ is approximately $75 \%$. Therefore we assign a probability of $75 \%$ to the opposite side of the square and a probability of $12.5 \%$ to each of the adjacent sides.

Step 9 tries to honour the coordination number distribution as a function of element radius. Generally, we find that the target coordination numbers are satisfied. If an element has its target coordination number reduced to zero then it cannot be chosen as an adjoining element in step 8 . However it could, by chance be linked to a new element which is positioned adjacent to another element. In this way, some coordination numbers are over-satisfied and some are under-satisfied. But this only affects a small percentage of the elements.

The process is complete when the minimum radius is reached and the element array is exhausted. At this point, not all lattice squares will be occupied and there may be some elements which are not connected to any others.

\subsection{Results}

Using the algorithm described above, we have constructed 2D stochastic networks representing three core plug samples.

The key input data for this process are the parameterised pore element radius distributions (Figure 8 and Table 2) plus the mean and standard deviation of the coordination number as a function of element radius (Figures 14 and 15). We also need the pore link radius distribution where the link radius is defined as the minimum of the two connected element radii. Analysis of the pore elements from SEM images, shows that the link radius distributions are very close to the element radius distributions with the parameter $r_{1}$ decreased by a factor of 0.7 to 0.8. Lastly, we define a rectangular domain which is the same size as the SEM image from which the statistics were obtained and define the total number of pore elements to be the same number as identified from the SEM image. This assumes that the SEM images are representative of the plug samples that were imaged.

Extracts from the generated stochastic networks are shown in Figure 19.

To validate the stochastic network algorithm, we have analysed some important statistics from the stochastic networks in comparison the pore element networks from SEM.

Firstly, Figure 20 compares the frequency distribution of coordination numbers for plug 130. We obtain a good match with the data from the SEM analysis. This is not surprising because the stochastic algorithm uses the element radius distribution and the mean coordination number as a function of radius therefore we should expect to reproduce the coordination number frequency distribution.

Secondly, we wish to analyse a measure of network connectivity. As the networks are $2 \mathrm{D}$ and the mean coordination number is around 2, we don't generally have large scale connectivity. However, smaller scale connectivity is determined by the clustering of elements. As a proxy for connectivity, we have analysed the number of element clusters of a given size as a function of the cluster size (Figure 21 ). This shows a power law behaviour with a scaling exponent of about 2 . The 


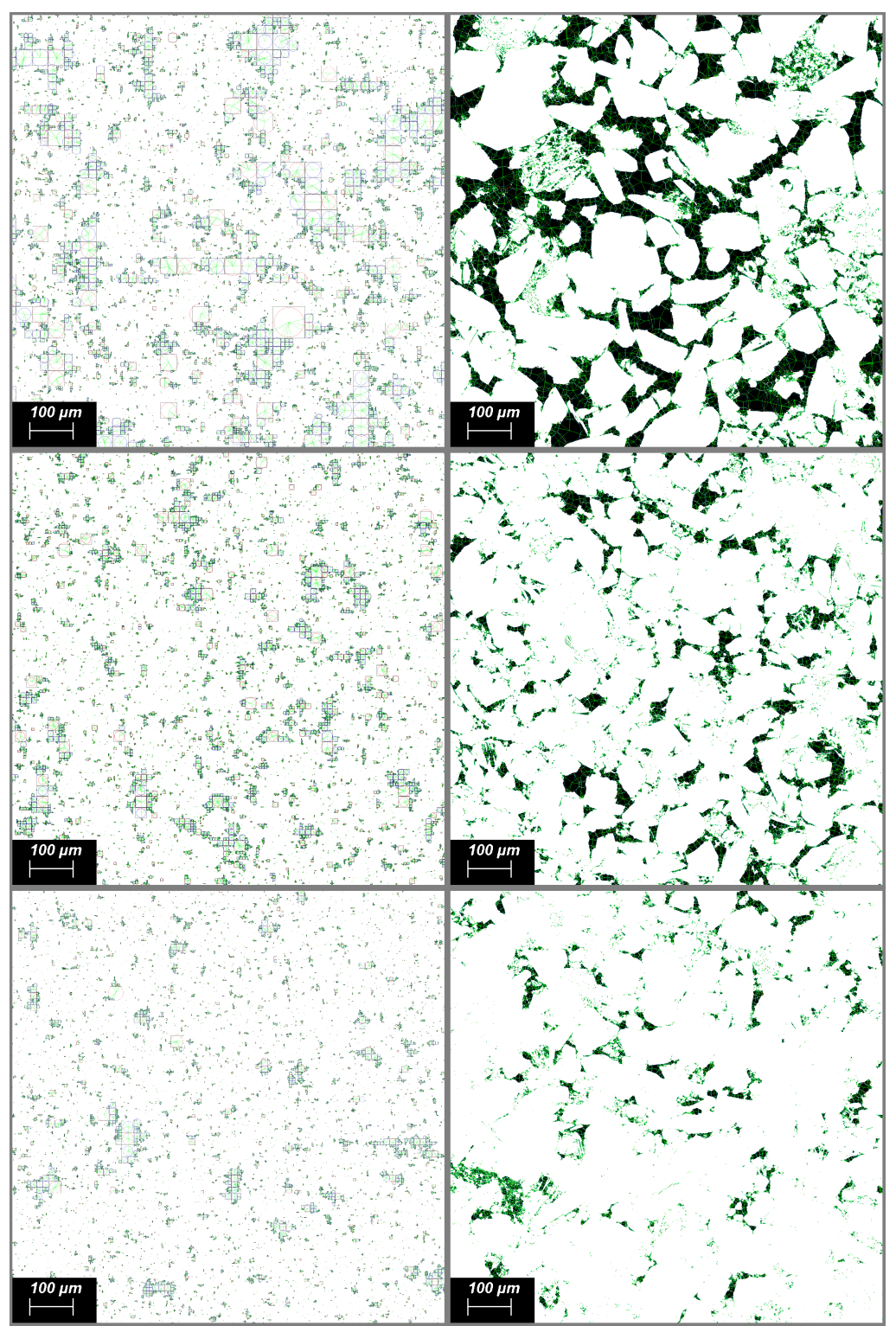

Fig. 19 Comparison between stochastic pore networks and pore element discretisation of SEM images: plug 44 (top), plug 130 (middle), plug 77 (bottom)

stochastic network shows a discrepancy for very small cluster sizes, in particular there are more isolated elements (cluster size $=1$ ) in the stochastic network. Otherwise, the stochastic network agrees well with the SEM image analysis. This gives us some encouragement that extending the procedure to $3 \mathrm{D}$ would generate networks with realistic spatial clustering.

Finally, we have examined the spatial distribution of pore elements. We divide the domain into square tiles or subregions such that the average number of elements 


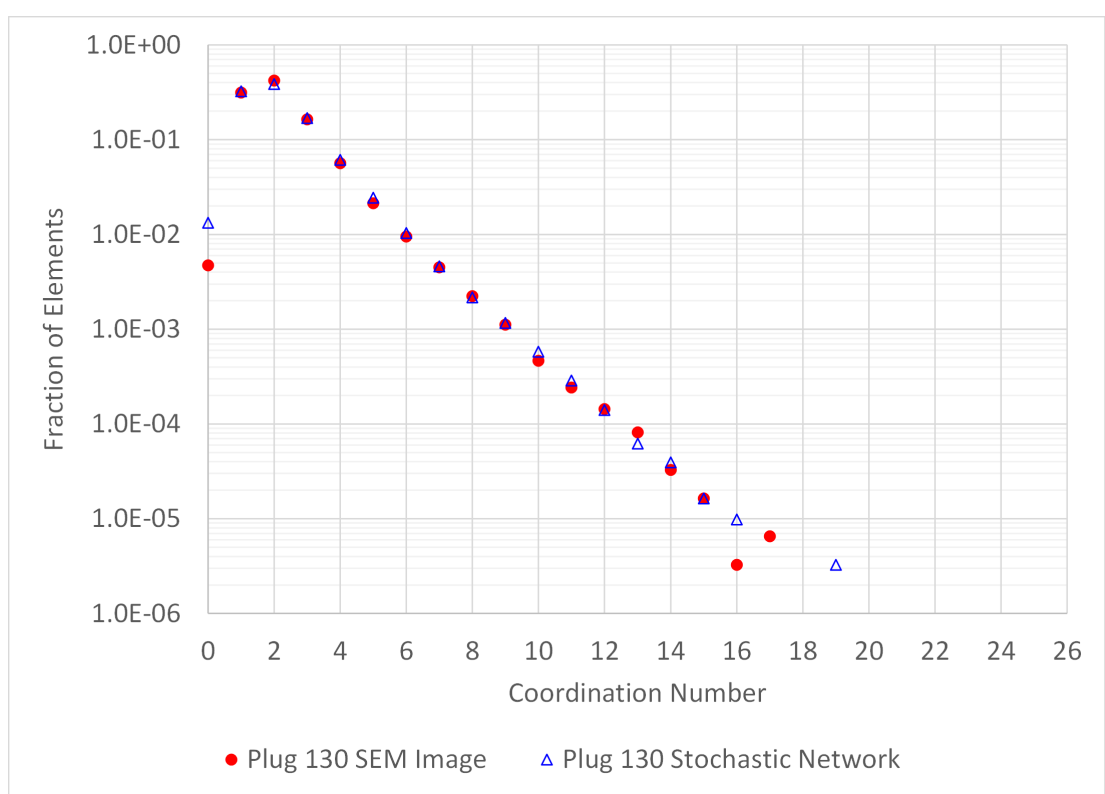

Fig. 20 Coordination number frequency distribution, comparison between stochastic pore network and SEM image network for plug 130

per subregion is 5 . Then we count the actual number of element in each subregion and plot the number of subregions containing a given number of elements (Figure 22 ). If the elements were uniformly randomly distributed, then this should be very close to a Poisson distribution with a mean of 5. Existing stochastic pore network algorithms presented by Idowu and Blunt (2008) and Jiang et al. (2012) assume that network nodes are uniformly randomly distributed in space, however Figure 22 shows that this is not a good assumption for complex sandstones. In particular, the number of subregions which contain zero elements is about 100 times larger than would be expected if the elements were uniformly randomly distributed. Our stochastic algorithm achieves a much more realistic spatial distribution of pore elements. This suggests that extending the algorithm to 3D will generate networks with a realistic spatial distribution of elements. Figure 22 shows that the algorithm under-predicts the number of subregions that contain a large number of elements. For example, the number of subregions containing 40 elements is under-predicted by a factor of about 20. The number of elements affected is approximately $0.3 \%$ of the total and we do not consider this to be significant.

\section{Conclusions}

We have use multi-scale SEM imaging to analyse the pore space geometry and topology of ten core samples from a sandstone reservoir. We use multi-scale imaging because it provides a much wider range of pore size information. The reservoir was buried to depths up to $3000 \mathrm{~m}$ and subjected to extensive diagenesis which has resulted in a composite pore space with a wide range in pore sizes from 0.01 


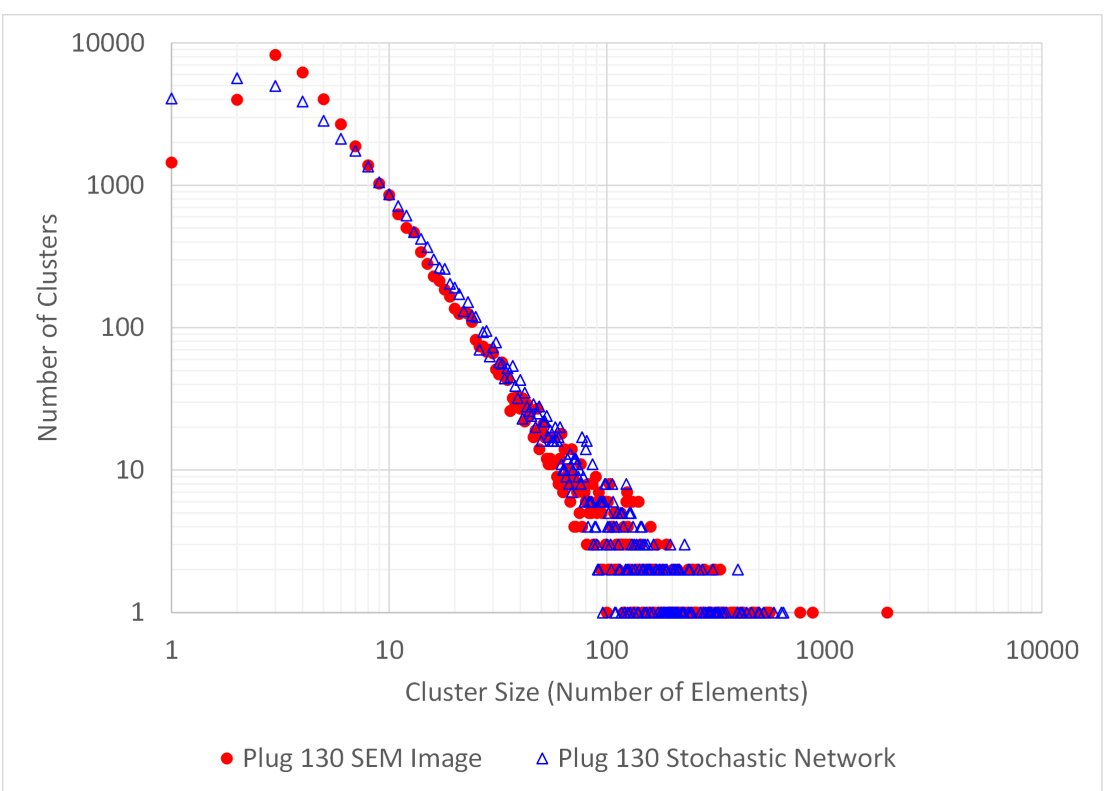

Fig. 21 Element cluster analysis, comparison between stochastic pore network and SEM image network for plug 130

$\mu \mathrm{m}$ to $100 \mu \mathrm{m}$. Although, we did not directly predict permeability and capillary pressure from the images, we found that rock property characteristics determined from $2 \mathrm{D}$ imaging using the proposed methods have a good relation with laboratory measured properties such as permeability and capillary pressure data.

Despite the combination of multiple complex processes influencing the pore space architecture, we find that pore size and connectivity measures such as coordination number show some regular character that can be represented by mathematical models.

The pore size distribution of each sample can be fitted to a modified power law (Pareto) distribution with an exponential cut-off at large radii. Based on analysis of 2D SEM images, the power law scaling index varies from 1.2 to 1.6.

We observe the frequency distribution of coordination numbers follows an exponential model where $P(Z) \propto \exp (-a Z)$ for some constant $a$. This is contrary to the power law model with scale free character proposed by Santiago (2008) for soil pore structures.

For a given pore size, the coordination number is approximately normally distributed. The mean and standard deviation of the coordination number are power law functions of pore radius, where the scaling exponent varies from 0.3 to 0.5.

We have presented a stochastic algorithm to generate a pore network in $2 \mathrm{D}$ based on pore size distributions and pore coordination number as a function of pore radius. The stochastic algorithm incorporates the concept of a weighted planar stochastic lattice which is a construction that naturally leads to scale-free character with power law behaviour. The algorithm honours the coordination number frequency distribution and the connectivity function. The algorithm also repro- 


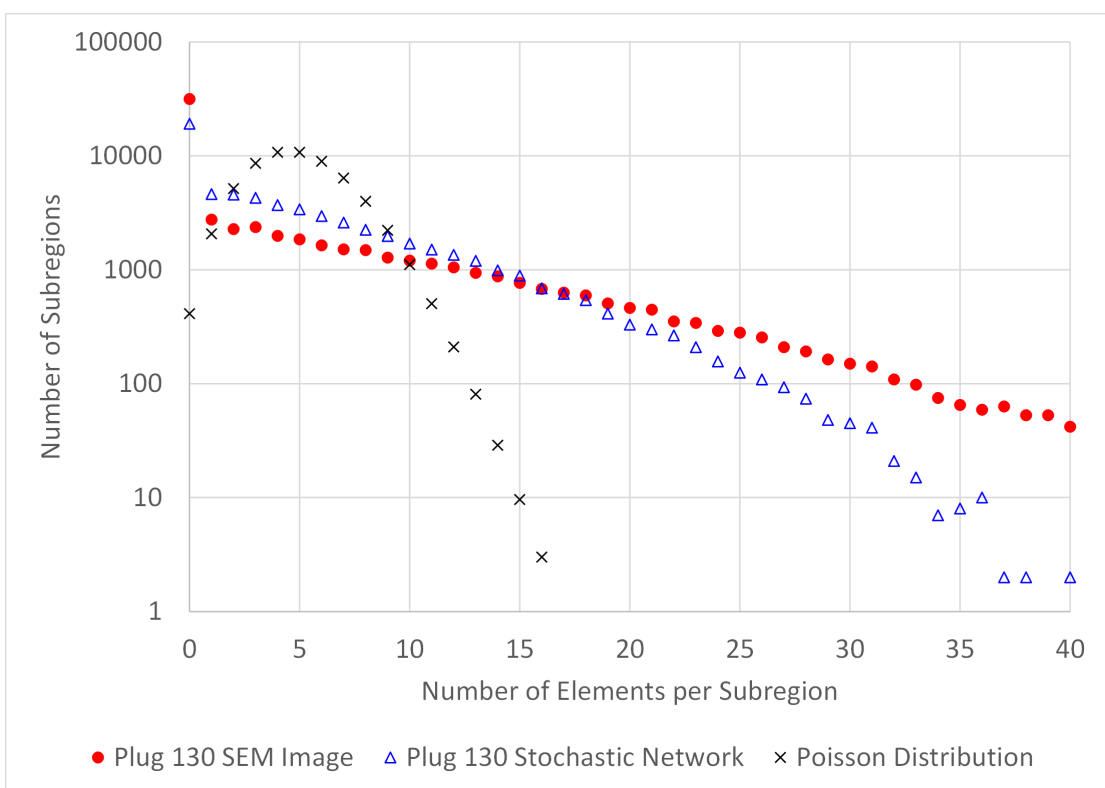

Fig. 22 Spatial distribution of elements, comparison between stochastic pore network and SEM image network for plug 130

duces the clustering of pore space elements observed in SEM images and close to a realistic spatial distribution of elements.

In further work, we plan to extend the pore space characterisation and stochastic algorithm to 3D. We will generate $3 \mathrm{D}$ stochastic pore network models based on $2 \mathrm{D}$ statistical information with $3 \mathrm{D}$ connectivity validated by $3 \mathrm{D}$ data. We intend to model relative permeability from quasi-static pore network simulations and compare with measured data. 


\section{Nomenclature}

$A_{\text {mean }} \quad$ Proportionality factor in the relationship between $Z_{\text {mean }}(r)$ and $r$

$A_{\text {stdev }} \quad$ Proportionality factor in the relationship between $Z_{\text {stdev }}(r)$ and $r$

$E \quad$ Euclidean distance

$E_{i} \quad$ Euclidean distance of pixel $i$

$n \quad$ Exponent of $\frac{r}{r_{1}}$ in the pore size probability distribution

$N_{e} \quad$ Number of elements

$N_{e}(r) \quad$ Number of elements with radius greater than $r$

$N_{e, t o t a l}$ Total number of elements

$N_{l} \quad$ Number of links

$N_{l}(r) \quad$ Number of links with radius greater than $r$

$N_{l, \text { total }}$ Total number of links

$r \quad$ Radius of element

$r_{i} \quad$ Radius of element $i$

$r_{\min } \quad$ Minimum element radius

$r_{\max } \quad$ Maximum element radius

$r_{0} \quad$ Pore size distribution parameter, near the minimum radius

$r_{1} \quad$ Pore size distribution parameter, near the maximum radius

$r_{x} \quad$ Characteristic radius of largest pores which control permeability

$Z \quad$ Coordination number

$Z_{i} \quad$ Coordination number of element $i$

$Z_{\text {mean }} \quad$ Mean coordination number

$Z_{\text {stdev }} \quad$ Standard deviation of the coordination number

$\alpha \quad$ Scaling index in the pore size probability distribution

$\beta \quad$ Scaling index in the relationship between $Z_{\text {mean }}(r)$ and $r$

$\phi \quad$ Porosity

$\chi \quad$ Connectivity function

Acknowledgements The authors would like to thank Anasuria Operating Company Ltd (AOC) for making available the rock samples for analysis.

SEM imaging was performed at the University of Aberdeen Centre for Electron Microscopy, Analysis and Characterisation (ACEMAC). We would like to thank John Still for invaluable assistance in the SEM imaging work.

The authors acknowledge the very helpful comments from three anonymous reviewers.

\section{References}

Akpokodje M, Melvin A, Churchill J, Burns S, Morris J, S K, Wakefield M, Ameerali R (2017) Regional study of controls on reservoir quality in the Triassic Skagerrak Formation of the Central North Sea. Proceedings of the 8th Petroleum Geology Conference pp 125-146, DOI 10.1144/PGC8.29

Barabasi A, Albert R (1999) Emergence of scaling in random networks. Science 286:509-512

Bashtani F, Maini B, Kantzas A (2016) Single-phase and two-phase flow properties of mesaverde tight sandstone formation; random-network modeling approach. Advances in Water Resources 94:174-184, DOI 10.1016/j.advwatres.2016.05.006 
Békri S, Laroche C, Vizika O (2005) Sca2005-35 pore network models to calculate transport and electrical properties of single or dual-porosity rocks. International Symposium of the Society of Core Analysts, Toronto

Blunt MJ, Bijeljic B, Dong H, Gharbi O, Iglauer S, Mostaghimi P, Paluszny A, Pentland C (2013) Pore-scale imaging and modelling. Advances in Water Resources 51:197-216, DOI 10.1016/j.advwatres.2012.03.003, URL http://dx.doi.org/10.1016/j.advwatres.2012.03.003

Borgefors G (1984) Distance transformations in arbitrary dimensions. Computer Vision, Graphics, \& Image Processing 27(3):321-345, DOI 10.1016/0734189X(84)90035-5

Bultreys T, Van Hoorebeke L, Cnudde V (2015) Multi-scale, micro-computed tomography-based pore network models to simulate drainage in heterogeneous rocks. Advances in Water Resources 78:36-49, DOI 10.1016/J.ADVWATRES.2015.02.003, URL https://www.sciencedirect.com/science/article/pii/S0309170815000299?via\%3Dihub

Bultreys T, De Boever W, Cnudde V (2016) Imaging and imagebased fluid transport modeling at the pore scale in geological materials: A practical introduction to the current state-of-the-art. EarthScience Reviews 155:93-128, DOI 10.1016/J.EARSCIREV.2016.02.001, URL https://www.sciencedirect.com/science/article/pii/S0012825216300150

Cannon SJC, Gowland S (1996) Facies controls on reservoir quality in the Late Jurassic Fulmar Formation, Quadrant 21, UKCS. Geology of the Humber Group; Central Graben and Moray Firth, UKCS 114(114):215-233, DOI 10.1144/GSL.SP.1996.114.01.10

Cook J, Goodwin L, Boutt D (2011) Systematic diagenetic changes in the grainscale morphology and permeability of a quartz-cemented quartz arenite. AAPG Bulletin 95(6)

De Boever E, Varloteaux1 C, FH N, Foubert A, Békri S, Youssef S, Rosenberg E (2012) Quantification and prediction of the 3d pore network evolution in carbonate reservoir rocks. Oil \& Gas Science and Technology 67(1):161-178, DOI $10.2516 /$ ogst/2011170

Freire-Gormaly M, Ellis J, MacLean HL, Bazylak A (2015) Pore structure characterization of indiana limestone and pink dolomite from pore network reconstructions. Oil and Gas Science and Technology 71(3), DOI 10.2516/ogst/2015004

Gowland S (1996) Facies characteristics and depositional models of highly bioturbated shallow marine siliciclastic strata: an example from the fulmar formation (late jurassic), uk central graben. Geology of the Humber Group; Central Graben and Moray Firth, UKCS 114:185-214

Hassan M, Hassan M, Pavel NI (2010) Scale-free network topology and multifractality in a weighted planar stochastic lattice. New Journal of Physics 12, DOI 10.1088/1367-2630/12/9/093045

Idowu N, Blunt M (2008) IPTC 12292 Pore-scale modelling of rate effects in waterflooding. Presented at the International Petroleum Technology Conference, Kuala Lumpur, Malaysia

Jiang Z, van Dijke MIJ, Wu K, Couples GD, Sorbie KS, Ma J (2012) Stochastic Pore Network Generation from 3D Rock Images. Transport in Porous Media 94(2):571-593, DOI 10.1007/s11242-011-9792-z

Jiang Z, Van Dijke MIJ, Sorbie KS, Couples GD (2013) Representation of multiscale heterogeneity via multiscale pore networks. Water Resources Research 
49(9):5437-5449, DOI 10.1002/wrcr.20304

Katz A, Thompson A (1987) A quantitative prediction of permeability in porous rock. Physical Review B 34:8179-8181, DOI 10.1103/PhysRevB.34.8179

Keehm Y, Mukerji T, Nur A (2004) Permeability prediction from thin sections: $3 \mathrm{~d}$ reconstruction and lattice-boltzmann flow simulation. Geophysical Research Letters 31(4), DOI 10.1029/2003GL018761

Lai J, Wang G, Cao J, Xiao C, Wang S, Pang X, Dai Q, He Z, Fan X, Yang L, Qin Z (2018) Investigation of pore structure and petrophysical property in tight sandstones. Marine and Petroleum Geology 91(November 2017):179-189, DOI 10.1016/j.marpetgeo.2017.12.024, URL https://doi.org/10.1016/j.marpetgeo.2017.12.024

Lindquist WB, Lee SM, Coker DA, Jones KW, Spanne P (1996) Medial axis analysis of void structure in three-dimensional tomographic images of porous media. Journal of Geophysical Research: Solid Earth 101(B4):8297-8310, DOI 10.1029/95JB03039, URL http://doi.wiley.com/10.1029/95JB03039

Manwart C, Torquato S, Hilfer R (2000) Stochastic reconstruction of sandstones. Phys Rev E 62:893-899, DOI 10.1103/PhysRevE.62.893, URL https://link.aps.org/doi/10.1103/PhysRevE.62.893

Naraghi ME, Spikes K, Srinivasan S (2017) 3D Reconstruction of Porous Media From a 2D Section and Comparisons of Transport and Elastic Properties. SPE Reservoir Evaluation \& Engineering 20(02):342-352, DOI 10.2118/180489-PA, URL http://www.onepetro.org/doi/10.2118/180489-PA

Okabe H, Blunt MJ (2007) Pore space reconstruction of vuggy carbonates using microtomography and multiple-point statistics. Water Resources Research 43(12):3-7, DOI 10.1029/2006WR005680

Øren P, Bakke S, Arntzen O (1998) SPE52052 Extending predictive capabilities to network models. SPE Journal

Otsu N (1979) A threshold selection method from gray-level histograms. IEEE Trans Sys, Man, Cyber 9(1):62-66, DOI 10.1109/TSMC.1979.4310076

Patzek TW (2001) Verification of a Complete Pore Network Simulator of Drainage and Imbibition. SPE Journal 6(02):144-156, DOI 10.2118/71310-PA, URL http://www.onepetro.org/doi/10.2118/71310-PA

Rabbani A, Jamshidi S, Salehi S (2014) An automated simple algorithm for realistic pore network extraction from microtomography images. Journal of Petroleum Science and Engineering 123:164-171, DOI 10.1016/j.petrol.2014.08.020, URL http://dx.doi.org/10.1016/j.petrol.2014.08.020

Rabbani A, Ayatollahi S, Kharrat R, Dashti N (2016) Estimation of 3-D pore network coordination number of rocks from watershed segmentation of a single 2-D image. Advances in Water Resources 94:264-277, DOI 10.1016/J.ADVWATRES.2016.05.020, URL https://www.sciencedirect.com/science/article/pii/S0309170816301543

Santiago A (2008) Multiscaling of porous soils as heterogeneous complex networks. Nonlinear Processes in Geophysics pp 893-902

Silin D, Patzek T (2006) Pore space morphology analysis using maximal inscribed spheres. Physica A 371:336-360, DOI 10.1016/j.physa.2006.04.048, URL https://ac.els-cdn.com/S037843710600464X/1-s2.0S037843710600464X-main.pdf?_tid=f07c7abd-565d-4042-a56cb3e390f9785f\&acdnat $=1525376481 \_2 c 971$ ad9e1ad35b710bf22cc6c29d4f7 
Tahmasebi P, Sahimi M (2012) Reconstruction of three-dimensional porous media using a single thin section. Phys Rev E 85, DOI 10.1103/PhysRevE.85.066709

Verscheure M, Fourno A, Chiles J (2012) Joint inversion of fracture model properties for co2 storage monitoring or oil recovery history matching. Oil \& Gas Science and Technology 67:221-235, DOI 10.2516/ogst/2011176

Vogel HJ, Roth K (2001) Quantitative morphology and network representation of soil pore structure. Advances in Water Resources 24(3-4):233-242, DOI 10.1016/S0309-1708(00)00055-5

Wu K, Nunan N, Crawford JW, Young IM, Ritz K (2004) An efficient Markov chain model for the simulation of heterogeneous soil structure. Soil Science Society of America Journal 68(2):346-351, DOI 10.2136/sssaj2004.0346, URL http://citeseerx.ist.psu.edu/viewdoc/download?doi=10.1.1.133.1586\&rep=rep1\&type=pdf

Yakubo K, Koroak D (2011) Scale-free networks embedded in fractal space. Physical Review E - Statistical, Nonlinear, and Soft Matter Physics 83(6), DOI 10.1103/PhysRevE.83.066111, 1009.0580

Youssef S, Rosenberg E, Gland N, Kenter J, Skalinski M, Vizika O (2007) Spe 111427 high resolution ct and pore-network models to assess petrophysical properties of homogeneous and heterogeneous carbonates. SPE/EAGE Reservoir Characterization Conference, Abu Dhabi, UAE

\section{A Downscaling Procedure}

Downscaling is a procedure we have employed to progressively incorporate information from high magnification (small field of view) SEM images into a low magnification (large field of view) SEM image. The resulting image has a high spatial resolution and a large field of view. The objective of this is to reduce the time required to characterise a complex pore space from SEM imaging. Images with a high spatial resolution and a large field of view can be acquired directly, but the acquisition time is typically several hours. The alternative is to acquire a sequence of images of progressively higher magnifications, with the same image resolution, then apply the downscaling algorithm to add information from the later (higher magnification) images into the first (lowest magnification) image. In particular, we use a sequence of images where each image has twice the magnification of the previous one. Each image can typically be acquired in a few minutes and therefore this process is significantly faster than acquiring a single high resolution image. The underlying assumption is that the pore structure detail (e.g. distribution of fine structure clays or cements) captured in the high magnification images is homogeneously distributed in the lowest magnification image.

Each stage of the downscaling process refines each pixel in the target binary image into $2 \times 2$ pixels based on the neighbourhood of $3 \times 3$ pixels. One stage is illustrated in Figure 23 .

Two images are required as input for each downscaling stage. The first is the source image which covers the required field of view but at a low spatial resolution. The second is a training image which has twice the spatial resolution of the source image but does not cover the field of view. The training image is coarsened by taking the arithmetic mean of each $2 \times 2$ pixel group to generate an image of the same spatial resolution as the source. The original and the coarsened training images are then segmented using the same threshold value to generate binary images. The two binary images are compared to calculate a probability matrix $\left\{p_{i j}\right\}$ where $p_{i j}$ is the probability of the fine scale $2 \times 2$ state $i$ given the coarse scale $3 \times 3$ state $j$ with $i=0,1,2,3, \ldots 2^{4}-1$ and $j=0,1,2,3, \ldots 2^{9}-1$. The probability $p_{i j}$ is conditional of the state, $j$, therefore we must satisfy:

$$
\sum_{i=0}^{15} p_{i j}=1 \quad \forall j
$$

For example, on the left in Figure 24 is part the coarse scale training image and the equivalent part of the fine scale training image is on the right. With pixels ordered in rows 


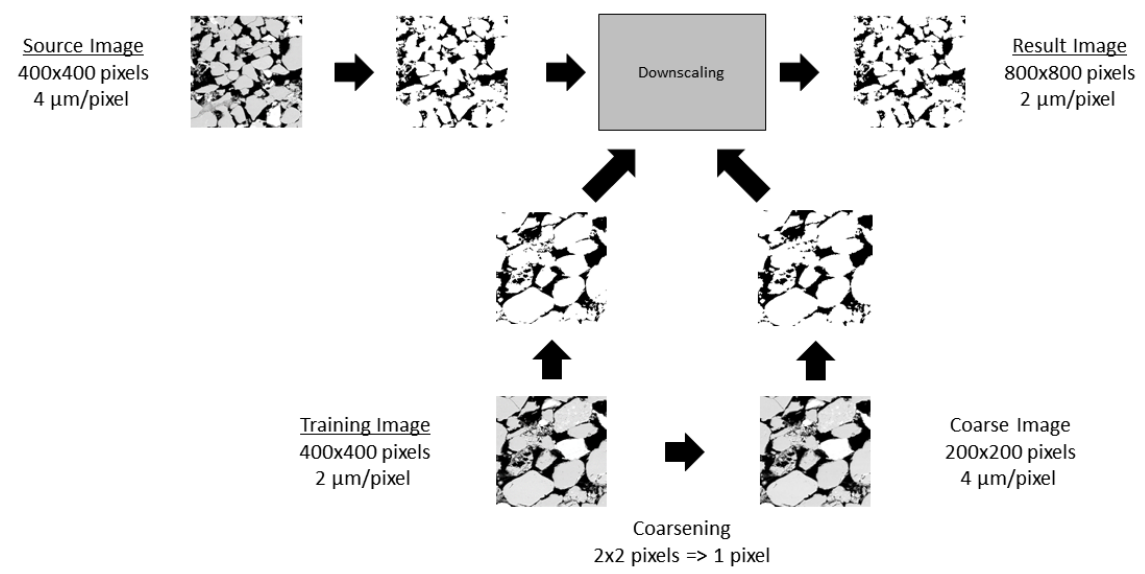

Fig. 23 One stage of the image downscaling process: the source image is downscaled using higher spatial resolution information from the training image
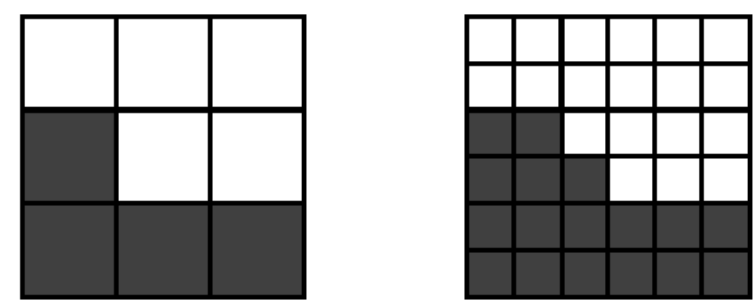

Fig. 24 Pixel states: part of the coarse training image showing state $j=39$ (left) and equivalent part of the fine training image showing state $i=2$ (right)

starting from the top left, taking black $=1$ and white $=0$, the left hand state, $j$, is 000100111 (binary) $=39$ (decimal) and the right hand state of the central $2 \times 2$ pixels, $i$, is 0010 (binary) $=2$ (decimal). The probability $p_{i j}=p_{2,39}$ is calculated from the number of occurrences of these patterns in the training images.

The probability matrix $\left\{p_{i j}\right\}$ has $2^{4} \times 2^{9}=8192$ elements, however the number of independent elements is only 1236 due to symmetry. The statistics for mirror symmetric and rotational symmetric states are combined in the algorithm.

If a certain state is not present in the training image then we use the value of the central pixel in the $3 \times 3$ group to determine all four of the pixels in the downscaled image. If the central pixel is black, then $p_{i j}=1$ for $i=15$ and $p_{i j}=0$ otherwise. If the central pixel is white, then $p_{i j}=1$ for $i=0$ and $p_{i j}=0$ otherwise.

Having calculated the probability matrix from the training image, we apply it to the source binary image to generate the result image. We calculate the state, $j$, of each $3 \times 3$ pixel neighbourhood in the source image and then randomly choose one of the states for the central pixel $2 \times 2$ refinement where the probability of choosing each state $i$ is $p_{i j}$.

Finally, we repeat the downscaling using a sequence of training images of progressively higher spatial resolution. The overall result is a single binary image with high resolution and a wide field of view. 